\title{
On the market viability under proportional transaction costs
}

\author{
Erhan Bayraktar $^{1} \quad$ Xiang $\mathrm{Yu}^{2}$
}

\begin{abstract}
${ }^{1}$ Department of Mathematics, University of Michigan, Ann Arbor, MI, USA

${ }^{2}$ Department of Applied Mathematics, The Hong Kong Polytechnic University, Hong Hum, Kowloon, Hong Kong

Correspondence

Erhan Bayraktar, Department of

Mathematics, University of Michigan, 530

Church Street, Ann Arbor, MI 48109, USA.

Email:erhan@umich.edu

\section{Funding information}

E. Bayraktar is supported in part by the National Science Foundation under Grant DMS-

1613170 and the Susan M. Smith Professorship.

$\mathrm{X}$. Yu is supported by the Start-Up Fund of the Hong Kong Polytechnic University under Grant 1-ZE5A.
\end{abstract}

\begin{abstract}
This paper studies the market viability with proportional transaction costs. Instead of requiring the existence of strictly consistent price systems as in the literature, we show that strictly consistent local martingale systems (SCLMS) can successfully serve as the dual elements such that the market viability can be verified. We introduce two weaker notions of no arbitrage conditions on market models named no unbounded profit with bounded risk (NUPBR) and no local arbitrage with bounded portfolios (NLABPs). In particular, we show that the NUPBR and NLABP conditions in the robust sense are equivalent to the existence of SCLMS for general market models. We also discuss the implications for the utility maximization problem.

\section{K E Y W O R D S}

utility maximization, market viability, numéraire portfolios, proportional transaction costs, (robust) no unbounded profit with bounded risk, (robust) no local arbitrage with bounded portfolios, strictly consistent local martingale systems
\end{abstract}

\section{1 | INTRODUCTION}

In the fundamental theorem of asset pricing with proportional transaction costs, consistent price systems (CPS) introduced by Jouini and Kallal (1995) and Cvitanić and Karatzas (1996) take the role of the dual elements instead of the equivalent (local) martingale measures. The CPS $(\tilde{S}, \mathbb{Q})$ is defined as follows:

Definition 1.1. Given the stock price $\left(S_{t}\right)_{t \in[0, T]}$ with transaction cost $\left(\lambda_{t}\right)_{t \in[0, T]}$ such that $0<\lambda_{t}<1$ a.s. for all $t \in[0, T]$, we call the pair $(\tilde{S}, \mathbb{Q})$ a CPS if

$$
\left(1-\lambda_{t}\right) S_{t} \leq \tilde{S}_{t} \leq\left(1+\lambda_{t}\right) S_{t}, \quad \mathbb{P} \text {-a.s. } \forall t \in[0, T],
$$


where $\left(\tilde{S}_{t}\right)_{t \in[0, T]}$ is a local martingale under $\mathbb{Q}$ and $\mathbb{Q} \sim \mathbb{P}$. Moreover, if we have

$$
\inf _{t \in[0, T]}\left(\lambda_{t} S_{t}-\left|S_{t}-\tilde{S}_{t}\right|\right)>0, \quad \mathbb{P} \text {-a.s. }
$$

the pair $(\tilde{S}, \mathbb{Q})$ is said to be a strictly CPS $(S C P S)$.

We should note that whether $\tilde{S}$ is required to be a local martingale or a true martingale in the above definition depends on the numéraire and numéraire-based admissibility of self-financing portfolios; see section 5 of Rásonyi and Schachermayer (2010) and Schachermayer (2015) for details. Sufficient conditions for the existence of a CPS for stock price processes with strictly positive and continuous paths have been extensively studied in the literature. One well-known example is the conditional full support condition proposed by Guasoni, Rásonyi, and Schachermayer (2008). Other related sufficient conditions are discussed in Bayraktar and Sayit (2010), Maris, Mbakop, and Sayit (2011), and Sayit and Viens (2011). Recently, for continuous price processes, Rásonyi and Schachermayer (2010) built the equivalence between the absence of arbitrage with general strategies for any small constant transaction $\operatorname{cost} \lambda>0$ and the existence of a CPS for any small transaction cost $\lambda>0$. Later, Guasoni, Lépinette, and Rásonyi (2012) investigated the general càdlàg processes and linked two equivalent assertions, i.e., the robust no free lunch with vanishing risk (NFLVR) for simple strategies and the existence of an SCPS.

On the other hand, in the market without transaction costs, the existing literature analyzed models that do not satisfy all the stringent requirements in the fundamental theorem of asset pricing. Compared to the NFLVR condition on terminal wealth originally defined by Delbaen and Schachermayer (1994), a weaker condition, which is called the no unbounded profit with bounded risk (NUPBR) condition in Karatzas and Kardaras (2007), serves as a reasonable substitute so that one can still solve the classical option hedging and utility maximization problems. Karatzas and Kardaras (2007), Becherer (2001), Christensen and Larsen (2007), Imkeller and Perkowski (2015), and Choulli, Deng, and Ma (2015) showed the equivalence between the NUPBR condition, the existence of a strictly positive local martingale deflator process, the existence of an optimal solution to the utility maximization problem, and the existence of a numéraire portfolio.

Motivated by these results obtained in frictionless markets, we aim to determine a similar minimal condition on the market with frictions under which the utility maximization problems still admit optimal solutions. However, due to the special nature of transaction costs, definitions of self-financing and admissibility of working portfolios differ from the usual ones on stochastic integrands for semimartingales. It is revealed in this paper that we need the stock price process $S$ to simultaneously meet two weaker conditions, i.e., the NUPBR and the no local arbitrage with bounded portfolios (NLABP) on liquidation value processes in the robust sense of Definition 2.7. It is worth noting that our NUPBR and NLABP conditions are still weaker than the NFLVR requirement in Guasoni et al. (2012) and even if both NUPBR and NLABP are satisfied, an arbitrage opportunity may still exist in the market. The main contribution of this paper is the equivalent characterization of the existence of a strictly consistent local martingale systems (SCLMS) $(\tilde{S}, Z)$, which is defined as follows.

Definition 1.2. Given the stock price $\left(S_{t}\right)_{t \in[0, T]}$ with transaction cost $\lambda_{t} \in(0.1)$ a.s. for all $t \in[0, T]$, we call a pair $(\tilde{S}, Z)$ a CLMS if $\tilde{S}$ is a semimartingale satisfying

$$
\left(1-\lambda_{t}\right) S_{t} \leq \tilde{S}_{t} \leq\left(1+\lambda_{t}\right) S_{t}, \quad \mathbb{P} \text {-a.s. }, \quad \forall t \in[0, T],
$$


and there exists a strictly positive local martingale $Z_{t}$ with $Z_{0}=1$ such that $\tilde{S}_{t} Z_{t}$ is a local martingale. We shall denote $\mathcal{Z}_{\text {loc }}(\lambda)$ the set of all CLMS with transaction cost $\left(\lambda_{t}\right)_{t \in[0, T]}$. Moreover, if

$$
\inf _{t \in[0, T]}\left(\lambda_{t} S_{t}-\left|S_{t}-\tilde{S}_{t}\right|\right)>0, \quad \mathbb{P} \text {-a.s. }
$$

we shall call the pair $(\tilde{S}, Z)$ an SCLMS and denote by $\mathcal{Z}_{l o c}^{s}(\lambda)$ the set of all SCLMS.

It is clear that the definition of a CLMS is a generalization of the classical CPS; i.e., any pair of CPS is a CLMS. However, the opposite is not necessarily true as $Z$ can be a strict local martingale. In Section 4, some examples of market models are presented, which demonstrate that an SCLMS may exist even when a CPS does not exist.

The second contribution of this paper is the result that shows that NUPBR and NLABP conditions in the robust sense guarantee the existence of a solution to the utility maximization problem defined on the terminal liquidation value. We also discuss the existence of a numéraire portfolio as a corollary. Therefore, NUPBR and NLABP conditions in the robust sense serve as sufficient conditions on the market viability in the sense that optimal portfolio problems admit solutions. Meanwhile, it is also shown that the market viability implies that the corresponding $S$ meets the NUPBR condition, although not in the robust sense, which illustrates that our market assumptions are minimal conditions to some extent.

To emphasize the mathematical differences between our setting and the frictionless market models in the literature, we discuss different types of arbitrage opportunities with transaction costs. In particular, we should point out that the NLABP condition in the main theorem is a new feature that appears for the first time. The construction of arbitrage opportunities in our setting with transaction costs is unique because the wealth process in frictionless market models has two counterparts, namely, the liquidation value process (see (2.2)) and the cost value process (see (3.11)). This difference leads to distinct arguments and proofs concerning the absence of arbitrage.

The rest of this paper is organized as follows: In Section 2, we introduce the market model with transaction costs and define the NUPBR and NLABP conditions on the terminal liquidation value. The equivalence between the NUPBR and NLABP conditions in the robust sense and the existence of the SCLMS is stated at the end of this section. The proof of the main theorem is given in Section 3. In Section 4, we discuss concrete examples of market models, for both continuous processes and jump processes, in which a CPS fails to exist, but we can find an SCLMS. Section 5 discusses the utility maximization problems under NUPBR and NLABP conditions in the robust sense. The discussion of various types of arbitrage opportunities and the comparison to the frictionless market models are provided in the first part of Section 6. In the second part of this section, we discuss our admissibility criterion.

\section{2 | SETUP AND THE MAIN RESULT}

The financial market consists of one risk-free bond $B$, normalized to be 1 , and one risky asset $S$. The given probability space $\left(\Omega, \mathcal{F},\left(\mathcal{F}_{t}\right)_{t \in[0, T]}, \mathbb{P}\right)$ is assumed to satisfy all the usual conditions of right continuity and completeness. $\mathcal{F}_{0}$ is assumed to be trivial. The following is a standing assumption that will hold in the rest of the paper:

Assumption 2.1. $\left(S_{t}\right)_{t \in[0, T]}$ is adapted to $\left(\mathcal{F}_{t}\right)_{t \in[0, T]}$ with strictly positive and locally bounded càdlàg paths. The transaction cost process $\left(\lambda_{t}\right)_{t \in[0, T]}$ is adapted to $\left(\mathcal{F}_{t}\right)_{t \in[0, T]}$ with càdlàg paths such that $\lambda_{t} \in(0,1)$ a.s. for all $t \in[0, T]$. 
We adopt the notion of self-financing admissible strategies defined in Schachermayer (2015):

Definition 2.2. A self-financing trading strategy starting with zero is a pair of predictable, finite variation processes $\left(\phi_{t}^{0}, \phi_{t}^{1}\right)_{t \in[0, T]}$ such that

(1) $\phi_{0}^{0}=\phi_{0}^{1}=0$,

(2) denoting by $\phi_{t}^{0}=\phi_{t}^{0, \uparrow}-\phi_{t}^{0, \downarrow}$ and $\phi_{t}^{1}=\phi_{t}^{1, \uparrow}-\phi_{t}^{1, \downarrow}$, the canonical decompositions of $\phi^{0}$ and $\phi^{1}$ into the difference of two increasing processes, starting at $\phi_{0}^{0, \uparrow}=\phi_{0}^{0, \downarrow}=\phi_{0}^{1, \uparrow}=\phi_{0}^{1, \downarrow}=0$, these processes satisfy

$$
\phi_{t}^{0, \uparrow} \leq \int_{0}^{t}\left(1-\lambda_{u}\right) S_{u} d \phi_{u}^{1, \downarrow}, \quad \phi_{t}^{0, \downarrow} \geq \int_{0}^{t}\left(1+\lambda_{u}\right) S_{u} d \phi_{u}^{1, \uparrow}, \quad \text { a.s. for } 0 \leq t \leq T,
$$

where the two integrals in (2.1) are defined as predictable Stieltjes integrals and

$$
\int_{s}^{t}\left(1-\lambda_{u}\right) S_{u} d \phi_{u}^{1, \downarrow} \triangleq \int_{s}^{t}\left(1-\lambda_{u}\right) S_{u} d \phi_{u}^{1, \downarrow, c}+\sum_{s<u \leq t}\left(1-\lambda_{u-}\right) S_{u-} \triangle \phi_{u}^{1, \downarrow}+\sum_{s \leq u<t}\left(1-\lambda_{u}\right) S_{u} \triangle_{+} \phi_{u}^{1, \downarrow}
$$

and

$$
\int_{S}^{t}\left(1+\lambda_{u}\right) S_{u} d \phi_{u}^{1, \uparrow} \triangleq \int_{s}^{t}\left(1+\lambda_{u}\right) S_{u} d \phi_{u}^{1, \uparrow, c}+\sum_{s<u \leq t}\left(1+\lambda_{u-}\right) S_{u-} \triangle \phi_{u}^{1, \uparrow}+\sum_{s \leq u<t}\left(1+\lambda_{u}\right) S_{u} \triangle_{+} \phi_{u}^{1, \uparrow}
$$

Here, $\triangle \phi_{t} \triangleq \phi_{t}-\phi_{t-}$ and $\triangle_{+} \phi_{t} \triangleq \phi_{t+}-\phi_{t}$. As discussed in Schachermayer (2015), because $S$ is càdlàg, we need to take care of both left and right jumps of the portfolio process $\phi$. In general, three values $\phi_{\tau-}, \phi_{\tau}$, and $\phi_{\tau+}$ can be different. If the stopping time $\tau$ is totally inaccessible, the predictability of $\phi$ implies that $\triangle \phi_{\tau}=0$ almost surely. But if the stopping time $\tau$ is predictable, it may happen that both $\triangle \phi_{\tau} \neq 0$ and $\triangle_{+} \phi_{\tau} \neq 0$.

In general, for any càdlàg process $X$ and predictable finite variation process $\phi$, the predictable Stieltjes integral above can be rewritten as

$$
\int_{0}^{t} X_{u} d \phi_{u}=\int_{0}^{t} X_{u} d \phi_{u-}-\sum_{s \leq t}\left(\phi_{s}-\phi_{s-}\right) \triangle X_{s}
$$

(See appendix A of Guasoni et al., 2012, for a detailed discussion on predictable Stieltjes integrals.)

At the initial time, we assume that the investor starts with the position $(x, 0)$ in bond and stock assets for the given constant $x \geq 0$. The trading strategy $\phi=\left(\phi^{0}, \phi^{1}\right)$ is called $x$-admissible if the liquidation value $V_{t}^{\text {liq,x }}$ satisfies

$$
V_{t}^{l i q, x}\left(\phi^{0}, \phi^{1}\right) \triangleq x+\phi_{t}^{0}+\left(\phi_{t}^{1}\right)^{+}\left(1-\lambda_{t}\right) S_{t}-\left(\phi_{t}^{1}\right)^{-}\left(1+\lambda_{t}\right) S_{t} \geq 0
$$

P-a.s. for $t \in[0, T]$. We shall denote $\mathcal{A}_{x}(\lambda)$ (short as $\mathcal{A}_{x}$ ) as the set of all $x$-admissible portfolios with the transaction cost $\left(\lambda_{t}\right)_{t \in[0, T]}$ and let $\mathcal{A}=\bigcup_{x \geq 0} \mathcal{A}_{x}$. Moreover, we will also denote $\mathcal{V}_{x}(\lambda)$ as the set of the terminal liquidation value $V_{T}^{\text {liq }}$ under the admissible portfolio $\left(\phi^{0}, \phi^{1}\right) \in \mathcal{A}_{x}(\lambda)$.

Parallel to the frictionless market, a weak no arbitrage condition can be defined via the boundedness in probability property of some target subset of $\mathbb{L}^{0}$. The following definition of NUPBR is analogous to that of Karatzas and Kardaras (2007). 
Definition 2.3. We say that $S$ admits an unbounded profit with bounded risk (UPBR) with the transaction cost $\lambda$ if there exists a sequence of admissible portfolios $\left(\phi^{0, n}, \phi^{1, n}\right)_{n \in \mathbb{N}}$ in $\mathcal{A}_{1}(\lambda)$ and the corresponding terminal liquidation value $\left(V_{T}^{l i q, 1}\left(\phi^{0, n}, \phi^{1, n}\right)\right)_{n \in \mathbb{N}}$ is unbounded in probability, i.e.,

$$
\lim _{m \rightarrow \infty} \sup _{n \in \mathbb{N}} \mathbb{P}\left(V_{T}^{l i q, 1}\left(\phi^{0, n}, \phi^{1, n}\right) \geq m\right)>0
$$

If no such sequence exists, we say that the stock price process $S$ satisfies the NUPBR condition under the transaction cost $\lambda$.

In order to provide the sufficient and necessary conditions on the existence of SCLMS, we also need to introduce another weak no arbitrage condition. To this end, let $\mathcal{A}_{x}^{\text {bd }}(\lambda)$ (short as $\mathcal{A}_{x}^{\text {bd }}$ ) denote the $x$-admissible bounded portfolios such that the position in the stock is uniformly bounded by some constant $M>0$ in the following sense:

$\mathcal{A}_{x}^{\mathrm{bd}}(\lambda) \triangleq\left\{\left(\phi^{0}, \phi^{1}\right):\left|\phi_{t}^{1}\right| \leq M, \mathbb{P}\right.$-a.s., $t \in[0, T]$ for some $M>0$ where $\left.\left(\phi^{0}, \phi^{1}\right) \in \mathcal{A}_{x}(\lambda)\right\}$.

Moreover, we denote $\mathcal{A}^{\text {bd }}=\bigcup_{x \geq 0} \mathcal{A}_{x}^{\text {bd }}$.

Definition 2.4. We say that $S$ satisfies NLABPs with the transaction cost $\lambda$ if there exists a sequence of stopping times $\tau_{n} \nearrow T$ as $n \rightarrow \infty$ such that for each $n \in \mathbb{N}$, we cannot find $\left(\phi^{0, n}, \phi^{1, n}\right) \in \mathcal{A}^{b d}(\lambda)$ that satisfies

$$
\mathbb{P}\left(V_{\tau_{n}}^{l i q, 0}\left(\phi^{0, n}, \phi^{1, n}\right) \geq 0\right)=1 \quad \text { and } \quad \mathbb{P}\left(V_{\tau_{n}}^{l i q, 0}\left(\phi^{0, n}, \phi^{1, n}\right)>0\right)>0 .
$$

It is noted that the NUPBR condition is defined on the set $\mathcal{A}_{x}$ for a fixed $x>0$, for instance $x=1$, which is consistent with the definition of the utility maximization problem with a fixed initial position. The NLABP condition is defined for all admissible portfolios on the set $\mathcal{A}$. However, these two definitions are consistent because if we have a sequence of portfolios in $\mathcal{A}_{1}$ that leads to UPBR; by rescaling, we also obtain UPBR for any $\mathcal{A}_{x}, x>0$.

For the completeness of the paper as well as the comparison between different concepts, the standard no arbitrage condition on liquidation values is provided next.

Definition 2.5. We say that $S$ admits arbitrage with the transaction costs $\lambda$ if there exits an admissible portfolio $\left(\phi^{0}, \phi^{1}\right) \in \mathcal{A}(\lambda)$ such that

$$
V_{0}^{l i q, 0}\left(\phi^{0}, \phi^{1}\right)=0, \quad \mathbb{P}\left(V_{T}^{l i q, 0}\left(\phi^{0}, \phi^{1}\right) \geq 0\right)=1 \quad \text { and } \quad \mathbb{P}\left(V_{T}^{l i q, 0}\left(\phi^{0}, \phi^{1}\right)>0\right)>0 .
$$

If no such portfolio exists, we say that the stock price process $S$ satisfies the NA condition under the transaction cost $\lambda$.

Remark 2.6. Comparing Definitions 2.4 and 2.5, it is clear that our NLABP is equivalent to the NA condition with bounded portfolios for a localizing sequence $\left\{\tau_{n}\right\}_{n \in \mathbb{N}}$. It is important to note that (NA) $\Rightarrow(N L A B P)$; however, (NLABP) does not imply (NA) in general. We refer the reader to a discussion and example 3.1 in section 3 of Choulli et al. (2015) noting that NA can hold locally but fail globally in frictionless markets. Moreover, the NUPBR condition and the NLABP condition may not imply each other. Given the assumption that NUPBR and NLABP are satisfied, we may still have arbitrage opportunities at time $T$ using some unbounded $x$-admissible portfolios $\left(\phi^{0}, \phi^{1}\right) \in \mathcal{A}_{x}$. The NFLVR condition in Guasoni et al. (2012) clearly implies both NUPBR and NLABP; therefore, we claim that our conditions are weaker assumptions on market models than the usual conditions in the literature. 
Some slightly stronger conditions are needed for the main result of this paper.

Definition 2.7. We say that $S$ satisfies the NUPBR and NLABP conditions with the transaction cost $\lambda$ in the robust sense if there exist another stock price process $\left(S_{t}^{\prime}\right)_{t \in[0, T]}$ and the transaction cost process $\left(\lambda_{t}^{\prime}\right)_{t \in[0, T]}$ satisfying Assumption 2.1 such that

$$
\inf _{t \in[0, T]}\left(\left(1+\lambda_{t}\right) S_{t}-\left(1+\lambda_{t}^{\prime}\right) S_{t}^{\prime}\right)>0 \text { a.s. and } \inf _{t \in[0, T]}\left(\left(1-\lambda_{t}^{\prime}\right) S_{t}^{\prime}-\left(1-\lambda_{t}\right) S_{t}\right)>0, \quad \text { a.s. }
$$

and the stock price process $S^{\prime}$ satisfies the NUPBR and NLABP conditions at the same time with the transaction cost $\lambda^{\prime}$. In particular, if we only consider the case where the stock price process $S^{\prime}$ satisfies the NUPBR condition with the transaction cost $\lambda^{\prime}$, we say that $S$ satisfies the robust NUPBR (RNUPBR) with the transaction cost $\lambda$.

As the main result of this paper, the following theorem provides the equivalence between NUPBR and NLABP conditions in the robust sense and the existence of SCLMS. Its proof is delivered in the next section.

Theorem 2.8. The following two assertions are equivalent:

(1) $S$ satisfies the NUPBR and NLABP conditions with the transaction cost $\lambda$ in the robust sense of Definition 2.7.

(2) There exists an $\operatorname{SCLMS}(\tilde{S}, Z)$ for the market with transaction cost $\lambda$, i.e., $\mathcal{Z}_{\text {loc }}^{s}(\lambda) \neq \emptyset$.

Remark 2.9. Compared to the frictionless markets in which we have the equivalence between the NUPBR condition on terminal wealth and the existence of local martingale deflators (see Karatzas and Kardaras, 2007), our equivalence characterization in the markets with transaction costs involves two conditions, i.e., NUPBR and NLABP. The self-financing and admissibility conditions in our framework are more restrictive than those in frictionless markets and different types of convergence are required in the two settings. For example, some convergence results for predictable Stieltjes integrals (theorem A.9 of Guasoni et al., 2012) and the integration by parts formula (proposition A.16 of Guasoni et al., 2012) play important roles in our proof; however, the literature in frictionless markets relies on the convergence results of stochastic integrals w.r.t. semimartingales.

Actually, in frictionless markets, the NLABP condition on wealth processes may always hold because either there is no local arbitrage (LA) for the wealth process $(H \cdot S)$ or there is an LA but the portfolio process $H$ is not necessarily bounded but is usually only required to be predictable and $S$-integrable. On the other hand, our stock price process $S$ is not necessarily a semimartingale and the liquidation value process lacks the supermartingale property, which is naturally possessed by each wealth process discounted by local martingale deflators in frictionless markets. For the equivalence between the NUPBR condition and the existence of local martingale deflators in models without transaction costs, the proof in Takaoka and Schweizer (2014) relies on the fact that the numéraire portfolio process is a supermartingale and a change of the numéraire and the proof in Karatzas and Kardaras (2007) is based on some probability characteristics of the semimartingale price process $S$. However, these results no longer hold in our setting. As discussed in Section 5, we do not expect the numéraire portfolio process to be a supermartingale. Some new ideas to support the proof of the equivalence in Theorem 2.8 are required. In particular, both NUPBR and NLABP are needed to guarantee our main result. 


\section{3 | PROOF OF THE THEOREM 2.8}

The proof of Theorem 2.8 is split into several steps. We first show that (2) $\Rightarrow(1)$.

\section{1 $\mid$ Proof of $(2) \Rightarrow(1)$}

Proposition 3.1. If there exists an $\operatorname{SCLMS}\left(\tilde{S}, Z_{t}\right) \in \mathcal{Z}_{\text {loc }}^{s}(\lambda)$, the stock price process $S$ satisfies the NUPBR and NLABP conditions with the transaction cost $\lambda$ in the robust sense.

Proof. Due to the existence of an $\operatorname{SCLMS}(\tilde{S}, Z)$ such that $\inf _{t \in[0, T]}\left(\lambda_{t} S_{t}-\left|S_{t}-\tilde{S}_{t}\right|\right)>0$ a.s., we can define $\xi_{t}=\inf _{s \in[0, t]}\left(\lambda_{S} S_{s}-\left|S_{S}-\tilde{S}_{S}\right|\right)$ and get that $\xi_{t}>0$ a.s. for all $t \in[0, T]$. Moreover, it is easy to see that

$$
\left(1-\lambda_{t}\right) S_{t}+\xi_{t} \frac{\lambda_{t}}{1+\lambda_{t}}<\tilde{S}_{t}<\left(1+\lambda_{t}\right) S_{t}-\xi_{t} \frac{\lambda_{t}}{1+\lambda_{t}} \text { a.s. } \forall t \in[0, T]
$$

as $0<\frac{\lambda_{t}}{1+\lambda_{t}}<1$. We can therefore choose $S_{t}^{\prime}=S_{t}$ for all $t \in[0, T]$ and $\lambda_{t}^{\prime}=\lambda_{t}-\xi_{t} \frac{\lambda_{t}}{\left(1+\lambda_{t}\right) S_{t}}$. First of all, it is straightforward to verify that

$$
\begin{aligned}
\inf _{t \in[0, T]}\left(\left(1-\lambda_{t}^{\prime}\right) S_{t}^{\prime}-\left(1-\lambda_{t}\right) S_{t}\right)>0, & \text { a.s. } \\
\text { and } \inf _{t \in[0, T]}\left(\left(1+\lambda_{t}\right) S_{t}-\left(1+\lambda_{t}^{\prime}\right) S_{t}^{\prime}\right)>0, & \text { a.s. }
\end{aligned}
$$

as well as $\inf _{t \in[0, T]}\left(\lambda_{t}^{\prime} S_{t}^{\prime}-\left|S_{t}^{\prime}-\tilde{S}_{t}\right|\right)>0$ a.s. Also, it holds that $0<\lambda_{t}^{\prime}<1$ for $t \in[0, T]$. To see this, it is sufficient to show that

$$
0<\frac{\xi_{t}}{\left(1+\lambda_{t}\right) S_{t}}<1 \text { a.s. } \forall t \in[0, T]
$$

which is a direct consequence of the definition of $\left(\xi_{t}\right)_{t \in[0, T]}$.

Thus, it is enough to prove that the smaller spread $\left[\left(1-\lambda_{t}^{\prime}\right) S_{t}^{\prime},\left(1+\lambda_{t}^{\prime}\right) S_{t}^{\prime}\right]$ satisfies the NUPBR and NLABP conditions with the transaction cost $\lambda^{\prime}$. Denote by $\mathcal{V}_{1}\left(\lambda^{\prime} ; S^{\prime}\right)$ the set of terminal liquidation value under 1 -admissible self-financing portfolios. We first show that $\mathcal{V}_{1}\left(\lambda^{\prime} ; S^{\prime}\right)$ is bounded in probability. To this end, let us first verify that

$$
\sup _{V_{T}^{\mathrm{liq}, 1} \in \mathcal{V}_{1}\left(\lambda^{\prime} ; S^{\prime}\right)} \mathbb{E}\left[V_{T}^{\mathrm{liq}, 1} Z_{T}\right]<\infty .
$$

$\tilde{S} Z$ is a local martingale by the definition of $\operatorname{SCLMS}(\tilde{S}, Z)$. We claim that for any admissible portfolio $\phi \in \mathcal{A}_{1}\left(\lambda^{\prime} ; S^{\prime}\right)$, the following holds:

$$
V_{t}^{\mathrm{liq}, 1}\left(\phi^{0}, \phi^{1}\right) \leq 1+\int_{0}^{t} \phi_{u}^{1} d \tilde{S}_{u} \text { a.s. } \forall t \in[0, T],
$$

where $\int_{0}^{t} \phi_{u}^{1} d \tilde{S}_{u}$ is interpreted as a stochastic integral. The liquidation value process can also be rewritten as

$$
V_{t}^{\mathrm{liq}, 1}\left(\phi^{0}, \phi^{1}\right)=1+\phi_{t}^{0}+\phi_{t}^{1} S_{t}^{\prime}-\lambda_{t}^{\prime}\left|\phi_{t}^{1}\right| S_{t}^{\prime}
$$


Using integration by parts (see proposition A.16 of Guasoni et al., 2012), we obtain

$$
\begin{aligned}
V_{t}^{\mathrm{liq}, 1}-\left(1+\int_{0}^{t} \phi_{u}^{1} d \tilde{S}_{u}\right) & =\phi_{t}^{0}+\phi_{t}^{1} S_{t}^{\prime}-\phi_{t}^{1} \tilde{S}_{t}+\int_{0}^{t} \tilde{S}_{u} d \phi_{u}^{1}-\lambda_{t}^{\prime}\left|\phi_{t}^{1}\right| S_{t}^{\prime} \\
& =\phi_{t}^{0}+\int_{0}^{t} \tilde{S}_{u} d \phi_{u}^{1}+\phi_{t}^{1}\left(S_{t}^{\prime}-\tilde{S}_{t}\right)-\lambda_{t}^{\prime}\left|\phi_{t}^{1}\right| S_{t}^{\prime},
\end{aligned}
$$

where the $\int_{0}^{t} \tilde{S}_{u} d \phi_{u}^{1}$ is a predictable Stieltjes integral. By (2.1) and the fact $\left(1-\lambda_{t}^{\prime}\right) S_{t}^{\prime}<\tilde{S}_{t}<(1+$ $\left.\lambda_{t}^{\prime}\right) S_{t}^{\prime}$ a.s. for all $t \in[0, T]$, we have that $\phi_{t}^{0}+\int_{0}^{t} \tilde{S}_{u} d \phi_{u}^{1} \leq 0$ and

$$
\phi_{t}^{1}\left(S_{t}^{\prime}-\tilde{S}_{t}\right)-\lambda_{t}^{\prime}\left|\phi_{t}^{1}\right| S_{t}^{\prime} \leq 0, \quad \text { a.s. } \forall t \in[0, T]
$$

which implies that $V_{t}^{\mathrm{liq}, 1}\left(\phi^{0}, \phi^{1}\right) \leq 1+\int_{0}^{t} \phi_{u}^{1} d \tilde{S}_{u}$ a.s. for $t \in[0, T]$.

Let $X_{t}=1+\int_{0}^{t} \phi_{u}^{1} d \tilde{S}_{u}$. The integration by parts formula yields that

$$
Z_{t} X_{t}=1+\int_{0}^{t}\left(X_{u-}-\phi_{u}^{1} \tilde{S}_{u-}\right) d Z_{u}+\int_{0}^{t} \phi_{u}^{1} d\left(\tilde{S}_{u} Z_{u}\right)
$$

Due to the Ansel-Stricker theorem (see Ansel \& Stricker, 1994), we get $Z_{t} X_{t}$ is a local martingale and therefore a supermartingale as $X_{t} \geq V_{t}^{\mathrm{liq}, 1}\left(\phi^{0}, \phi^{1}\right) \geq 0$.

Therefore, it follows that

$$
\mathbb{E}\left[V_{T}^{\text {liq, } 1} Z_{T}\right] \leq \mathbb{E}\left[X_{T} Z_{T}\right] \leq X_{0} Z_{0}=1
$$

The fact that the right-hand side is independent of the choice of $V_{T}^{\text {liq,1 }}$ yields that (3.1) holds true.

By (3.1) and the fact that $Z_{t}$ is strictly positive for all $t \in[0, T]$ and hence $Z_{T}>0$, P-a.s., lemma 3.2 of Imkeller and Perkowski (2015) implies that the set $\mathcal{V}_{1}\left(\lambda^{\prime} ; S^{\prime}\right)$ is bounded in probability. Therefore, the conclusion holds that $S^{\prime}$ satisfies the NUPBR condition.

On the other hand, to show that $S^{\prime}$ satisfies the NLABP condition is straightforward. Due to the fact that $\tilde{S} Z$ and $Z$ are local martingales, there exists a localizing sequence $\left\{\tau_{n}\right\}_{n \in \mathbb{N}}$ such that $\tilde{S}_{t \wedge \tau_{n}} Z_{t \wedge \tau_{n}}$ and $Z_{t \wedge \tau_{n}}$ are true martingales. For the same sequence $\left\{\tau_{n}\right\}_{n \in \mathbb{N}}$, suppose that for some $n \in \mathbb{N}$, there exists some bounded admissible portfolio $\left(\phi^{0}, \phi^{1}\right) \in \mathcal{A}^{\mathrm{bd}}(\lambda)$ such that (2.5) holds for the stopping time $\tau_{n}$. Define the probability measure $\mathbb{Q} \sim \mathbb{P}$ by $\frac{d \mathbb{Q}}{d \mathbb{P}}=Z_{\tau_{n}}$. It follows that $\tilde{S}_{t \wedge \tau_{n}}$ is a martingale under $\mathbb{Q}$. Moreover, as $\left|\phi_{t}^{1}\right| \leq M$ a.s. for some $M>0$, the stochastic integral $\int_{0}^{t \wedge \tau_{n}} \phi_{u}^{1} d \tilde{S}_{u}$ is a true martingale under $\mathbb{Q}$. Therefore, we can deduce that

$$
\mathbb{E}^{\mathbb{Q}}\left[V_{\tau_{n}}^{\mathrm{liq}, 0}\left(\phi^{0}, \phi^{1}\right)\right] \leq \mathbb{E}^{\mathbb{Q}}\left[\int_{0}^{\tau_{n}} \phi_{u}^{1} d \tilde{S}_{u}\right]=0 .
$$

However, this is a contradiction to $\mathbb{Q}\left(V_{\tau_{n}}^{\text {liq,0 }}\left(\phi^{0}, \phi^{1}\right) \geq 0\right)=1$ and $\mathbb{Q}\left(V_{\tau_{n}}^{\text {liq,0 }}\left(\phi^{0}, \phi^{1}\right)>0\right)>0$ by the fact that $\mathbb{Q} \sim \mathbb{P}$ as well as (2.5). Therefore, we obtain a sequence of stopping times $\tau_{n} \nearrow T$ that satisfies Definition 2.4 and $S^{\prime}$ satisfies the NLABP condition. 


\section{$3.2 \mid$ Proof of $(1) \Rightarrow$ (2)}

The proof of this direction requires more preparation. To begin with, it is noted that the set $\mathcal{V}_{x}(\lambda)$ itself is not convex. We thereby shall consider its solid hull defined by

$$
\mathcal{C}(1) \triangleq\left\{V \in \mathbb{L}_{+}^{0}: V \leq V_{T}^{\mathrm{liq}, 1} \in \mathcal{V}_{1}(\lambda)\right\} .
$$

Clearly, $\mathcal{C}(x)=\left\{V \in \mathbb{L}_{+}^{0}: V \leq V_{T}^{\text {liq }, x} \in \mathcal{V}_{x}(\lambda)\right\}=x \mathcal{C}(1)$ and $\mathcal{C}(x)$ is convex and solid.

Lemma 3.2. If the stock price process $\left(S_{t}\right)_{t \in[0, T]}$ satisfies $R N U P B R$ with the transaction cost $\left(\lambda_{t}\right)_{t \in[0, T]}$, the set $\left\{\left\|\phi^{1}\right\|_{T}:\left(\phi^{0}, \phi^{1}\right) \in \mathcal{A}_{1}(\lambda ; S)\right\}$, where $\left\|\phi^{1}\right\|_{T}$ denotes the total variation of $\phi^{1}$ on $[0, T]$, is bounded in probability.

Proof. Let $\left(\lambda^{\prime}, S^{\prime}\right)$ be as in Definition 2.7. For any $\phi=\left(\phi^{0}, \phi^{1}\right) \in \mathcal{A}_{1}(\lambda ; S) \subset \mathcal{A}_{1}\left(\lambda^{\prime} ; S^{\prime}\right)$, we have

$$
\begin{aligned}
0 \leq & V_{t}^{\mathrm{liq}, 1 ; S, \lambda}\left(\phi^{0}, \phi^{1}\right) \leq 1+\int_{0}^{t}\left(1-\lambda_{u}\right) S_{u} d \phi_{u}^{1, \downarrow}-\int_{0}^{t}\left(1+\lambda_{u}\right) S_{u} d \phi_{u}^{1, \uparrow}+\phi_{t}^{1} S_{t}-\lambda_{t}\left|\phi_{t}^{1}\right| S_{t} \\
= & 1+\int_{0}^{t}\left(1-\lambda_{u}^{\prime}\right) S_{u}^{\prime} d \phi_{u}^{1, \downarrow}-\int_{0}^{t}\left(1+\lambda_{u}^{\prime}\right) S_{u}^{\prime} d \phi_{u}^{1, \uparrow}+\phi_{t}^{1} S_{t}^{\prime}-\lambda_{t}^{\prime}\left|\phi_{t}^{1}\right| S_{t}^{\prime} \\
& -\int_{0}^{t}\left(\left(1-\lambda_{u}^{\prime}\right) S_{u}^{\prime}-\left(1-\lambda_{u}\right) S_{u}\right) d \phi_{u}^{1, \downarrow}-\int_{0}^{t}\left(\left(1+\lambda_{u}\right) S_{u}-\left(1+\lambda_{u}^{\prime}\right) S_{u}^{\prime}\right) d \phi_{u}^{1, \uparrow} \\
& +\phi_{t}^{1}\left(S_{t}-S_{t}^{\prime}\right)-\left|\phi_{t}^{1}\right|\left(\lambda_{t} S_{t}-\lambda_{t}^{\prime} S_{t}^{\prime}\right) \text { a.s. } \forall t \in[0, T] .
\end{aligned}
$$

Let us define $\xi_{t}=\inf _{s \leq t}\left(\left(1-\lambda_{s}^{\prime}\right) S_{s}^{\prime}-\left(1-\lambda_{s}\right) S_{s}\right)$ and $\eta_{t}=\inf _{s \leq t}\left(\left(1+\lambda_{s}\right) S_{s}-\left(1+\lambda_{s}^{\prime}\right) S_{s}^{\prime}\right)$. Because $\phi_{t}^{1}\left(S_{t}-S_{t}^{\prime}\right)-\left|\phi_{t}^{1}\right|\left(\lambda_{t} S_{t}-\lambda_{t}^{\prime} S_{t}^{\prime}\right)<0$ for all $t \in[0, T]$, it follows from (3.5) that

$$
\begin{aligned}
\left(\xi_{T} \wedge \eta_{T}\right)\left\|\phi^{1}\right\|_{T} & \leq \int_{0}^{T}\left(\left(1-\lambda_{u}^{\prime}\right) S_{u}^{\prime}-\left(1-\lambda_{u}\right) S_{u}\right) d \phi_{u}^{1, \downarrow}+\int_{0}^{T}\left(\left(1+\lambda_{u}\right) S_{u}-\left(1+\lambda_{u}^{\prime}\right) S_{u}^{\prime}\right) d \phi_{u}^{1, \uparrow} \\
& \leq 1+\int_{0}^{T}\left(1-\lambda_{u}^{\prime}\right) S_{u}^{\prime} d \phi_{u}^{1, \downarrow}-\int_{0}^{T}\left(1+\lambda_{u}^{\prime}\right) S_{u}^{\prime} d \phi_{u}^{1, \uparrow}+\phi_{T}^{1} S_{T}^{\prime}-\lambda_{T}^{\prime}\left|\phi_{T}^{1}\right| S_{T}^{\prime} \\
& =V_{T}^{\mathrm{liq}, 1 ; S^{\prime}, \lambda^{\prime}} \text { a.s. }
\end{aligned}
$$

The assumption that $S$ satisfies the RNUPBR condition on $[0, T]$ yields that $\mathcal{V}_{1}\left(\lambda^{\prime} ; S^{\prime}\right)$ is bounded in probability. By assumption $\xi_{T}>0$ and $\eta_{T}>0$ a.s. and lemma 3.1 of Guasoni (2002), we obtain that the set $\left\{\|\phi\|_{T}, \phi \in \mathcal{A}_{1}(\lambda ; S)\right\}$ is bounded in probability.

The proof of the following result is also crucial in establishing the existence of the optimal solution of the utility maximization problem as well as the existence of a numéraire portfolio in Section 5.

Proposition 3.3. If $\left(S_{t}\right)_{t \in[0, T]}$ satisfies the RNUPBR with transaction cost $\left(\lambda_{t}\right)_{t \in[0, T]}$, the set $\mathcal{C}(1)$ is closed under convergence in probability.

Proof. Take a sequence $V_{T}^{n} \in \mathcal{C}(1)$ such that $V_{T}^{n} \rightarrow \hat{V}_{T}$ in probability. By passing to a subsequence, we can assume without loss of generality that $V_{T}^{n} \rightarrow \hat{V}_{T}$ a.s. The proof boils down to proving that $\hat{V}_{T} \in \mathcal{C}(1)$. Consider now a sequence $X^{n} \in \mathcal{V}_{1}$ satisfying $V_{T}^{n} \leq X_{T}^{n}$ a.s. By the definition of $\mathcal{V}_{1}$, there exist a sequence $\left(\phi^{0, n}, \phi^{1, n}\right) \in \mathcal{A}_{1}$ and $X_{t}^{n}=1+\phi_{t}^{0, n}+\phi_{t}^{1, n} S_{t}-\lambda_{t}\left|\phi_{t}^{1, n}\right| S_{t}$ for all $t \in[0, T]$. 
Lemma 3.2 states that the set $\left\{\left\|\phi^{1}\right\|_{T}:\left(\phi^{0}, \phi^{1}\right) \in \mathcal{A}_{1}\right\}$ is bounded in probability. Due to lemma B.4 of Guasoni et al. (2012), we can deduce that there exists a sequence of forward convex combinations $\theta^{n} \in \operatorname{conv}\left(\phi^{1, n}, \phi^{1, n+1}, \ldots\right)$ such that $\theta^{n}$ converges pointwise to a predictable and finite variation process $\hat{\phi}^{1}$ such that the sequence $\left\|\theta^{n}\right\|$ also converges to $\left\|\hat{\phi}^{1}\right\|$ pointwise. The latter convergence implies that the sequence $\left(\left\|\theta^{n}\right\|_{t}\right)_{n \in \mathbb{N}}$ converges to $\left\|\hat{\phi}^{1}\right\|_{t}$ in probability for each $t \in[0, T]$, which, in turn, leads to the fact that the set $\left\{\left\|\theta^{n}\right\|_{t}\right\}_{n \in \mathbb{N}}$ is bounded in probability for each $t \in[0, T]$. Similar to the proof of lemma 4.3 of Guasoni (2002), we can write

$$
\left\{\limsup _{n \rightarrow \infty}\left\|\theta^{n}\right\|_{t}>M\right\}=\left\{\liminf _{n \rightarrow \infty}\left\|\theta^{n}\right\|_{t}>M\right\}=\bigcup_{k} \bigcap_{n \geq k}\left\{\left\|\theta^{n}\right\|_{t}>M\right\},
$$

which gives that

$$
\mathbb{P}\left(\limsup _{n \rightarrow \infty}\left\|\theta^{n}\right\|_{t}>M\right)=\mathbb{P}\left(\bigcup_{k} \bigcap_{n \geq k}\left\{\left\|\theta^{n}\right\|_{t}>M\right\}\right) \leq \sup _{n} \mathbb{P}\left(\left\|\theta^{n}\right\|_{t}>M\right) .
$$

For each $t \in[0, T]$, as the set $\left\{\left\|\theta^{n}\right\|_{t}\right\}_{n \in \mathbb{N}}$ is bounded in probability, we can obtain that

$$
\lim _{M \rightarrow \infty} \mathbb{P}\left(\limsup _{n \rightarrow \infty}\left\|\theta^{n}\right\|_{t}>M\right)=0,
$$

which implies that $\limsup _{n \rightarrow \infty}\left\|\theta^{n}\right\|_{t}<\infty$ a.s. and hence $\sup _{n \geq 1}\left\|\theta^{n}\right\|_{t}<\infty$ a.s. for $t \in[0, T]$. As a result, we can apply the assertion (iii) of theorem A.9 of Guasoni et al. (2012), to obtain the pointwise convergence of predictable Stieltjes integrals

$$
\lim _{n \rightarrow \infty} \int_{0}^{t} S_{u} d \theta_{u}^{n}=\int_{0}^{t} S_{u} d \hat{\phi}_{u}^{1}
$$

which holds for any càdlàg process $S$.

Using the same sequence of convex combinations in the definition of $\theta^{n}$, without loss of generality, we can consider $X^{n}$ as the resulting process after the forward convex combinations. Similarly, we define $\theta^{0, n}=\operatorname{conv}\left(\phi^{0, n}, \phi^{0, n+1}, \ldots\right)$ following the same convex combinations. It follows that

$$
X_{t}^{n} \leq 1+\theta_{t}^{0, n}+\theta_{t}^{n} S_{t}-\lambda_{t}\left|\theta_{t}^{n}\right| S_{t} \text {, a.s. } \forall t \in[0, T] .
$$

Therefore, we obtain that

$$
\begin{aligned}
X_{t}^{n} & \leq 1+\int_{0}^{t}\left(1-\lambda_{u}\right) S_{u} d \theta_{u}^{n, \downarrow}-\int_{0}^{t}\left(1+\lambda_{u}\right) S_{u} d \theta_{u}^{n, \uparrow}+\theta_{t}^{n} S_{t}-\lambda_{t}\left|\theta_{t}^{n}\right| S_{t} \\
& =1+\int_{0}^{t} S_{u} d \theta_{u}^{n}-\int_{0}^{t} \lambda_{u} S_{u} d\left\|\theta^{n}\right\|_{u}+\theta_{t}^{n} S_{t}-\lambda_{t}\left|\theta_{t}^{n}\right| S_{t}, \text { a.s. } \forall t \in[0, T] .
\end{aligned}
$$

Due to (iv) of theorem A.9 of Guasoni et al. (2012), the lower semicontinuity property holds in the sense that

$$
\int_{0}^{t} S_{u} d\left\|\hat{\phi}^{1}\right\|_{u} \leq \liminf _{n \rightarrow \infty} \int_{0}^{t} S_{u} d\left\|\theta^{n}\right\|_{u}, \text { a.s. } \forall t \in[0, T]
$$


Letting $n \rightarrow \infty$ in (3.7) and using (3.6) and (3.8), we can obtain

$$
\begin{aligned}
\lim _{n \rightarrow \infty} X_{t}^{n} & \leq 1+\int_{0}^{t} S_{u} d \hat{\phi}_{u}^{1}-\int_{0}^{t} \lambda_{u} S_{u} d\left\|\hat{\phi}^{1}\right\|_{u}+\hat{\phi}_{t}^{1} S_{t}-\lambda_{t}\left|\hat{\phi}_{t}^{1}\right| S_{t} \\
& =1+\int_{0}^{t}\left(1-\lambda_{u}\right) S_{u} d \hat{\phi}_{u}^{1, \downarrow}-\int_{0}^{t}\left(1+\lambda_{u}\right) S_{u} d \hat{\phi}_{u}^{1, \uparrow}+\hat{\phi}_{t}^{1} S_{t}-\lambda_{t}\left|\hat{\phi}_{t}^{1}\right| S_{t} \\
& =1+\hat{\phi}_{t}^{0}+\hat{\phi}_{t}^{1} S_{t}-\lambda_{t}\left|\hat{\phi}_{t}^{1}\right| S_{t}, \text { a.s. }
\end{aligned}
$$

for $t \in[0, T]$, where $\hat{\phi}_{t}^{0} \triangleq \int_{0}^{t}\left(1-\lambda_{s}\right) S_{s} d \hat{\phi}_{s}^{1, \downarrow}-\int_{0}^{t}\left(1+\lambda_{s}\right) S_{s} d \hat{\phi}_{s}^{1, \uparrow}$ for all $t \in[0, T]$. By definition, $\left(\hat{\phi}^{0}, \hat{\phi}^{1}\right)$ is a self-financing portfolio. Moreover, because $X_{t}^{n} \geq 0$ a.s. for all $t \in[0, T]$ and $n \in \mathbb{N}$, we can see that $\left(\hat{\phi}^{0}, \hat{\phi}^{1}\right) \in \mathcal{A}_{1}$. It follows from $V_{T}^{n} \rightarrow \hat{V}_{T}$ a.s. that

$$
\hat{V}_{T} \leq \hat{X}_{T} \in \mathcal{V}_{1} \text {, a.s. }
$$

where

$$
\hat{X}_{t} \triangleq 1+\hat{\phi}_{t}^{0}+\hat{\phi}_{t}^{1} S_{t}-\lambda_{t}\left|\hat{\phi}_{t}^{1}\right| S_{t}, \quad \forall t \in[0, T]
$$

Therefore, $\hat{V} \in \mathcal{C}(1)$, which completes the proof.

For the proof of the next few results, we shall consider the stopped liquidation values starting with zero initial position and $x$-admissible portfolios, $x \geq 0$. For any stopping time $\tau$, let us define the convex and solid set

$$
\mathbf{C}^{\tau}(x) \triangleq\left\{V \in \mathbb{L}^{0}: V \leq V_{\tau}^{\text {liq }, 0}\left(\phi^{0}, \phi^{1}\right) \text { for }\left(\phi^{0}, \phi^{1}\right) \in \mathcal{A}_{x}\right\}
$$

and

$$
\mathbf{C}^{\tau}=\bigcup_{x \geq 0} \mathbf{C}^{\tau}(x)
$$

Fix the initial position $(x, 0)$. For each self-financing portfolio $\left(\phi^{0}, \phi^{1}\right)$, we call the process $V^{\operatorname{cost}, x}\left(\phi^{0}, \phi^{1}\right)$ the cost value to enter the portfolio position $\left(\phi^{0}, \phi^{1}\right)$, which is defined as

$$
V_{t}^{\text {cost }, x}\left(\phi^{0}, \phi^{1}\right) \triangleq x+\phi_{t}^{0}+\left(\phi_{t}^{1}\right)^{+}\left(1+\lambda_{t}\right) S_{t}-\left(\phi_{t}^{1}\right)^{-}\left(1-\lambda_{t}\right) S_{t}, \quad t \in[0, T] .
$$

For the same pair of self-financing portfolio process $\left(\phi^{0}, \phi^{1}\right)$, it follows that

$$
V_{t}^{\mathrm{cost}, x}\left(\phi^{0}, \phi^{1}\right)=V_{t}^{\mathrm{liq}, x}\left(\phi^{0}, \phi^{1}\right)+2 \lambda_{t} S_{t}\left|\phi_{t}^{1}\right|, \quad t \in[0, T] .
$$

Remark 3.4. To understand the financial interpretation of the cost value process $V^{\text {cost }, x}$, let us consider two investors $A$ and B. Starting from the initial time, investor A holds the initial position $(x, 0)$ in two accounts and uses the portfolio $\left(\phi^{0}, \phi^{1}\right)$ during the time period $[0, T]$. On the other hand, investor $B$ does not follow the market until an intermediate time $t$. At time $t$, investor $B$ wants the same position $\left(\phi_{t}^{0}, \phi_{t}^{1}\right)$ that $A$ holds in two accounts. The process $V_{t}^{\text {cost }, x}$ represents the total cash amount that $B$ needs to enter this position pair at time t. It is noted that B needs enough cash to cover not only the bank and stock accounts, but also the transaction cost necessary to replicate the position $\phi_{t}^{1}$.

In the frictionless market with the semimartingale stock price process $S$, the condition of selffinancing portfolios can be relaxed to predictable and $S$-integrable portfolios. In these circumstances, 
the definition of liquidation value processes $\left(V_{t}^{l i q, x}\right)_{t \in[0, T]}$ and the definition of cost value processes $\left(V_{t}^{\text {cost }, x}\right)_{t \in[0, T]}$ coincide and they are both equivalent to the definition of wealth processes $\left(X_{t}\right)_{t \in[0, T]}=$ $\left(x+\left(\phi^{1} \cdot S\right)_{t}\right)_{t \in[0, T]}$.

For a fixed level $M>0$ and a stopping time $\tau \leq T$, let us consider a subset $\mathbf{C}_{M}^{\tau}(x)$ of the set $\mathbf{C}^{\tau}(x)$ defined by

$$
\mathbf{C}_{M}^{\tau}(x) \triangleq\left\{V: V \leq V_{\tau}^{\text {liq, } 0}\left(\phi^{0}, \phi^{1}\right) \quad \text { where } \quad\left(\phi^{0}, \phi^{1}\right) \in \mathcal{A}_{x} \quad \text { and } \quad\left|\phi_{t}^{1}\right| \leq M, \mathbb{P} \text {-a.s. on } \llbracket 0, \tau \rrbracket\right\} .
$$

We also define $\mathbf{C}_{M}^{\tau} \triangleq \bigcup_{x \geq 0} \mathbf{C}_{M}^{\tau}(x)$. It is clear that the set $\mathbf{C}_{M}^{\tau}$ is not empty, as the constant zero is an element. For $M$ large enough, proposition A.11 of Guasoni et al. (2012), which gives that $\left(\phi^{0}, \phi^{1}\right)$ is locally bounded, implies that the set $\mathbf{C}_{M}^{\tau}$ contains some nonzero elements.

Lemma 3.5. Assume that the stock price process $\left(S_{t}\right)_{t \in[0, T]}$ satisfies the NLABP condition with the transaction cost $\left(\lambda_{t}\right)_{t \in[0, T]}$. Given a fixed level $M>0$ large enough and the same sequence of stopping times $\tau_{n} \nearrow T$ as in Definition 2.4, for each $n \in \mathbb{N}$, we have that for any $x>0$ and any $x$-admissible portfolio $\left(\phi^{0}, \phi^{1}\right) \in \mathcal{A}_{x}$ with $\left|\phi_{t}^{1}\right| \leq M$, P-a.s. on $\llbracket 0, \tau_{n} \rrbracket$, we have

$$
V_{\tau_{n}}^{l i q, 0}\left(\phi^{0}, \phi^{1}\right) \geq-a \text { a.s. } \Rightarrow V_{t}^{\text {cost }, 0}\left(\phi^{0}, \phi^{1}\right) \geq-a \text {, a.s. } \forall t \leq \tau_{n},
$$

for any $0<a<x$.

Proof. Suppose not, i.e., for some $n \in \mathbb{N}$ and the corresponding stopping time $\tau_{n}$, there exists $a>$ 0 and a fixed pair of $x$-admissible portfolio $\left(\phi^{0}, \phi^{1}\right) \in \mathcal{A}_{x}$ such that $\left|\phi_{t}^{1}\right| \leq M$ for $0 \leq t \leq \tau_{n}$ and $V_{\tau_{n}}^{\text {liq, } 0}\left(\phi^{0}, \phi^{1}\right) \in \mathbf{C}_{M}^{\tau_{n}}(x)$ and

$$
V_{\tau_{n}}^{\mathrm{liq}, 0}\left(\phi^{0}, \phi^{1}\right) \geq-a, \text { a.s. }
$$

but for some stopping time $s$ s.t. $\mathbb{P}\left(s<\tau_{n}\right)=1$, we have

$$
\mathbb{P}\left(V_{s}^{\text {liq, }, 0}\left(\phi^{0}, \phi^{1}\right)+2 \lambda_{s} S_{s}\left|\phi_{s}^{1}\right|<-a\right)>0 .
$$

Denote the set $D \triangleq\left\{V_{s}^{\text {liq, } 0}\left(\phi^{0}, \phi^{1}\right)+2 \lambda_{s} S_{s}\left|\phi_{s}^{1}\right|<-a\right\}$.

Based on the fixed bounded pair $\left(\phi^{0}, \phi^{1}\right)$, we shall construct a new pair of self-financing portfolio $\left(\bar{\phi}^{0}, \bar{\phi}^{1}\right) \in \mathcal{A}_{x^{\prime}}$ with the initial position $\left(x^{\prime}, 0\right)$ for some $x^{\prime}>0$. To this end, given the previous $\tau_{n}$ and $s$, the new pair $\left(\bar{\phi}^{0}, \bar{\phi}^{1}\right)$ is defined by

$$
\begin{aligned}
& \bar{\phi}_{t}^{1} \triangleq \phi_{t}^{1} \mathbf{1}_{\rrbracket s, \tau_{n} \rrbracket} \mathbf{1}_{D}, \\
& \bar{\phi}_{t}^{0} \triangleq\left(\left(\phi_{t}^{0}-V_{s}^{\operatorname{cost}, 0}\left(\phi^{0}, \phi^{1}\right)\right) \mathbf{1}_{\rrbracket s, \tau_{n} \rrbracket}+\left(V_{\tau_{n}}^{\text {liq, }, 0}\left(\phi^{0}, \phi^{1}\right)-V_{s}^{\operatorname{cost}, 0}\left(\phi^{0}, \phi^{1}\right)\right) \mathbf{1}_{\rrbracket \tau_{n}, T \rrbracket}\right) \mathbf{1}_{D} .
\end{aligned}
$$

To check that $\left(\bar{\phi}^{0}, \bar{\phi}^{1}\right)$ is self-financing, we observe that

$$
\begin{gathered}
\triangle \bar{\phi}_{t}^{0}=\triangle \bar{\phi}_{t}^{1, \uparrow}=\triangle \bar{\phi}_{t}^{1, \downarrow}=0, \quad 0 \leq t \leq s, \\
\triangle_{+} \bar{\phi}_{t}^{0}=\triangle_{+} \bar{\phi}_{t}^{1, \uparrow}=\triangle_{+} \bar{\phi}_{t}^{1, \downarrow}=0, \quad 0 \leq t<s .
\end{gathered}
$$

At the time $s$, we have

$$
\triangle_{+} \bar{\phi}_{s}^{1, \uparrow}=\left(\phi_{s}^{1}\right)^{+} \mathbf{1}_{D} \text { and } \quad \triangle_{+} \bar{\phi}_{s}^{1, \downarrow}=\left(\phi_{s}^{1}\right)^{-} \mathbf{1}_{D}
$$


As for $\bar{\phi}^{0}$, we can deduce that

$$
\begin{aligned}
\triangle_{+} \bar{\phi}_{s}^{0} & =\left(\phi_{s}^{0}-\phi_{s}^{0}-\left(1+\lambda_{s}\right) S_{s}\left(\phi_{s}^{1}\right)^{+}+\left(1-\lambda_{s}\right) S_{S}\left(\phi_{s}^{1}\right)^{-}\right) \mathbf{1}_{D} \\
& =\left(-\left(1+\lambda_{s}\right) S_{s}\left(\phi_{s}^{1}\right)^{+}+\left(1-\lambda_{s}\right) S_{S}\left(\phi_{s}^{1}\right)^{-}\right) \mathbf{1}_{D} \\
& =-\left(1+\lambda_{s}\right) S_{S} \triangle_{+} \bar{\phi}_{s}^{1, \uparrow}+\left(1-\lambda_{s}\right) S_{s} \triangle_{+} \bar{\phi}_{s}^{1, \downarrow} .
\end{aligned}
$$

For the stochastic interval $\rrbracket s, \tau_{n} \llbracket$, it is clear that

$$
d \bar{\phi}_{t}^{1}=d \phi_{t}^{1} \quad \text { and } \quad d \bar{\phi}_{t}^{0}=d \phi_{t}^{0}
$$

Therefore, $\left(\bar{\phi}^{0}, \bar{\phi}^{1}\right)$ is self-financing on $\rrbracket s, \tau_{n} \llbracket$ as $\left(\phi^{0}, \phi^{1}\right)$ is self-financing on $\rrbracket s, \tau_{n} \llbracket$.

At last, at the stopping time $\tau_{n}$, it is clear that

$$
\triangle_{+} \bar{\phi}_{\tau_{n}}^{1, \uparrow}=\left(\phi_{\tau_{n}}^{1}\right)^{-} \mathbf{1}_{D} \quad \text { and } \quad \triangle_{+} \bar{\phi}_{\tau_{n}}^{1, \downarrow}=\left(\phi_{\tau_{n}}^{1}\right)^{+} \mathbf{1}_{D}
$$

We therefore also have

$$
\begin{aligned}
\triangle_{+} \bar{\phi}_{\tau_{n}}^{0} & =\left(V_{\tau_{n}}^{\mathrm{liq}, 0}\left(\phi^{0}, \phi^{1}\right)-V_{s}^{\mathrm{cost}, 0}\left(\phi^{0}, \phi^{1}\right)-\left(\phi_{\tau_{n}}^{0}-V_{s}^{\mathrm{cost}, 0}\left(\phi^{0}, \phi^{1}\right)\right)\right) \mathbf{1}_{D} \\
& =\left(V_{\tau_{n}}^{\mathrm{liq}, 0}\left(\phi^{0}, \phi^{1}\right)-\phi_{\tau_{n}}^{0}\right) \mathbf{1}_{D} \\
& =\left(\left(1-\lambda_{\tau_{n}}\right) S_{\tau_{n}}\left(\phi_{\tau_{n}}^{1}\right)^{+}-\left(1+\lambda_{\tau_{n}}\right) S_{\tau_{n}}\left(\phi_{\tau_{n}}^{1}\right)^{-}\right) \mathbf{1}_{D} \\
& =\left(1-\lambda_{\tau_{n}}\right) S_{\tau_{n}} \triangle_{+} \bar{\phi}_{s}^{1, \downarrow}-\left(1+\lambda_{\tau_{n}}\right) S_{\tau_{n}} \triangle_{+} \bar{\phi}_{\tau_{n}}^{1, \uparrow}
\end{aligned}
$$

For the stochastic interval $\rrbracket \tau_{n}, T \rrbracket$, we clearly know that $d \bar{\phi}_{t}^{1}=d \bar{\phi}_{t}^{0}=0$. Putting all pieces together, we arrive at the conclusion that the pair $\left(\bar{\phi}^{0}, \bar{\phi}^{1}\right)$ is self-financing. Moreover, $\left|\phi^{1}\right| \leq M$ on $\rrbracket s, \tau_{n} \rrbracket$ implies that $\left(\bar{\phi}^{0}, \bar{\phi}^{1}\right)$ is also bounded in the sense that $\left|\bar{\phi}_{t}^{1}\right| \leq M$, P-a.s., $t \in[0, T]$ for some $M>0$.

In order to show that $\left(\bar{\phi}^{0}, \bar{\phi}^{1}\right)$ is also admissible, we first define the constant $x^{\prime}=x-a>0$ and notice that for the stochastic interval $\llbracket 0, s \rrbracket$,

$$
V_{t}^{\text {liq, } x^{\prime}}\left(\bar{\phi}^{0}, \bar{\phi}^{1}\right)=x^{\prime}>0 \text {, a.s. }
$$

Now, on the stochastic interval $\rrbracket s, \tau_{n} \llbracket$, we have

$$
\begin{aligned}
V_{t}^{\mathrm{liq}, x^{\prime}}\left(\bar{\phi}^{0}, \bar{\phi}^{1}\right) & =x^{\prime}+\bar{\phi}_{t}^{0}+\left(1-\lambda_{t}\right) S_{t}\left(\bar{\phi}_{t}^{1}\right)^{+}-\left(1+\lambda_{t}\right) S_{t}\left(\bar{\phi}_{t}^{1}\right)^{-} \\
& =x^{\prime}+\left(V_{t}^{\mathrm{liq}, 0}\left(\phi^{0}, \phi^{1}\right)-V_{s}^{\operatorname{cost}, 0}\left(\phi^{0}, \phi^{1}\right)\right) \mathbf{1}_{D} \\
& \geq x^{\prime}+(-x+a) \mathbf{1}_{D} \geq x^{\prime}-x+a=0, \text { a.s. }
\end{aligned}
$$


where we recall that $\left(\phi^{0}, \phi^{1}\right) \in \mathcal{A}_{x}^{\text {bd }}$. Similarly, on the stochastic interval $\llbracket \tau_{n}, T \rrbracket$, we have

$$
\begin{aligned}
V_{t}^{\mathrm{liq}, x^{\prime}}\left(\bar{\phi}^{0}, \bar{\phi}^{1}\right) & =x^{\prime}+\bar{\phi}_{t}^{0}+\left(1-\lambda_{t}\right) S_{t}\left(\bar{\phi}_{t}^{1}\right)^{+}-\left(1+\lambda_{t}\right) S_{t}\left(\bar{\phi}_{t}^{1}\right)^{-} \\
& =x^{\prime}+\left(V_{\tau_{n}}^{\mathrm{liq}, 0}\left(\phi^{0}, \phi^{1}\right)-V_{s}^{\mathrm{cost}, 0}\left(\phi^{0}, \phi^{1}\right)\right) \mathbf{1}_{D} \\
& >x^{\prime}+(-a+a) \mathbf{1}_{D}=x^{\prime}, \text { a.s. }
\end{aligned}
$$

In particular, we have $V_{\tau_{n}}^{\text {liq, } x^{\prime}}\left(\bar{\phi}^{0}, \bar{\phi}^{1}\right)>x^{\prime}$ a.s., so the bounded portfolio $\left(\bar{\phi}^{0}, \bar{\phi}^{1}\right) \in \mathcal{A}_{x^{\prime}}^{\text {bd }}$ leads to an arbitrage opportunity for the liquidation value process locally at the stopping time $\tau_{n}$. We obtain a contradiction to the NLABP condition in Definition 2.4. As a consequence, the implication (3.13) holds.

Lemma 3.6. Assume that the stock price process $\left(S_{t}\right)_{t \in[0, T]}$ satisfies the NLABP condition with the transaction cost $\left(\lambda_{t}\right)_{t \in[0, T]}$. Given a fixed level $M>0$ large enough and the same sequence of stopping times $\tau_{n} \nearrow T$ as in Definition 2.4, for each $n \in \mathbb{N}$, we have $\mathbf{C}_{M}^{\tau_{n}} \cap \mathbb{L}_{+}^{\infty}=\{0\}$.

Proof. According to the definition of $\mathbf{C}_{M}^{\tau_{n}}$, it is equivalent to prove that $\mathbf{C}_{M}^{\tau_{n}}(x) \cap \mathbb{L}_{+}^{\infty}=\{0\}$ for any $x>$ 0 . Suppose that the above claim does not hold. Then, for some $n \in \mathbb{N}$ and the corresponding stopping time $\tau_{n}$, there exists a pair of portfolios $\left(\phi^{0}, \phi^{1}\right) \in \mathcal{A}_{x}$ for some $x>0$ such that $\left|\phi_{t}^{1}\right| \leq M$ for $0 \leq t \leq \tau_{n}$ and

$$
\mathbb{P}\left(V_{\tau_{n}}^{\text {liq }, 0}\left(\phi^{0}, \phi^{1}\right) \geq 0\right)=1 \quad \text { and } \quad \mathbb{P}\left(V_{\tau_{n}}^{\text {liq }, 0}\left(\phi^{0}, \phi^{1}\right)>0\right)>0
$$

Obviously, this is a contradiction to the definition of the NLABP condition that completes the proof.

As the stock price $\left(S_{t}\right)_{t \in[0, T]}$ is locally bounded, there is an increasing sequence of constants $\alpha(n) \nearrow+\infty$ and an increasing sequence of stopping times $\rho_{n} \nearrow T$ such that $S_{t} \leq \alpha(n)$ on the stochastic interval $\llbracket 0, \rho^{n} \rrbracket$. Given the sequence of stopping times $\left\{\tau_{n}\right\}_{n \in \mathbb{N}}$ in Definition 2.4, let us consider the new sequence of stopping times

$$
\bar{\tau}_{0} \triangleq 0, \quad \bar{\tau}_{n} \triangleq \tau_{n} \wedge \rho_{n}, \quad \text { for } n \in \mathbb{N} .
$$

Remark 3.7. It is easy to verify that the statements in Lemmas 3.5 and 3.6 holds for the sequence of stopping times $\left(\bar{\tau}_{n}\right)_{n \in \mathbb{N}}$ defined by (3.14) and that the stock price process $S$ is bounded up to $\bar{\tau}_{n}$ for each $n \in \mathbb{N}$.

Lemma 3.8. Assume that the stock price process $S$ satisfies the RNUPBR and NLABP conditions with the transaction cost $\lambda$. Given the fixed level $M>0$ large enough and the sequence of stopping times $\left\{\bar{\tau}_{n}\right\}_{n \in \mathbb{N}}$ defined in (3.14), for each $n \in \mathbb{N}$, we have that $\mathbf{C}_{M}^{\bar{\tau}_{n}}$ is Fatou closed; i.e., if there exists a sequence $\left(V^{m}\right)_{m \in \mathbb{N}}$ in $\mathbf{C}_{M}^{\bar{\tau}_{n}}$ such that $V^{m} \geq-$ for some $a>0$ and $V^{m}$ converges to some $\mathcal{F}_{\bar{\tau}_{n}}$-measurable random variable $V, \mathbb{P}$-a.s., then we have that $V \in \mathbf{C}_{M}^{\bar{\tau}_{n}}$.

Proof. Given the sequence $\left(V^{m}\right)_{m \in \mathbb{N}} \subset \mathbf{C}_{M}^{\bar{\tau}_{n}}$, for each $m$, there exists a portfolio $\left(\phi^{0, m}, \phi^{1, m}\right) \in \mathcal{A}_{x(m)}$ for some $x(m)>0$ (we write $x(m)$ to emphasize the dependence of $x$ on $m$ ) and $\left|\phi_{t}^{1, m}\right| \leq M$ for the fixed bound level $M$ and stopping time $\bar{\tau}_{n}$ such that $V^{m} \leq V_{\bar{\tau}_{n}}^{\text {liq,0 }}\left(\phi^{0, m}, \phi^{1, m}\right)$. Lemma 3.5 and Remark 
3.7 guarantee that $V_{\bar{\tau}_{n}}^{\text {liq, } 0}\left(\phi^{0, m}, \phi^{1, m}\right) \geq-a$ implies that $V_{t}^{\text {cost }, 0}\left(\phi^{0, m}, \phi^{1, m}\right) \geq-a$ for $0 \leq t \leq \bar{\tau}_{n}$. In particular, for each $m>0$, we get

$$
V_{t}^{\mathrm{liq}, 0}\left(\phi^{0, m}, \phi^{1, m}\right) \geq-2 \lambda_{t} S_{t}\left|\phi_{t}^{1, m}\right|-a \geq-2 \alpha(n) M-a, \text { a.s. for } 0 \leq t \leq \bar{\tau}_{n} .
$$

Therefore, we can apply Lemma 3.2 with the initial position $x=2 \alpha(n) M+a$ and obtain that the set $\left\{\left\|\phi^{1}\right\|_{\bar{\tau}_{n}}:\left(\phi^{0}, \phi^{1}\right) \in \mathcal{A}_{2 \alpha(n) M+a}(\lambda ; S)\right\}$ is also bounded in probability. Moreover, by choosing the initial position $x=2 \alpha(n) M+a$ and time interval $0 \leq t \leq \bar{\tau}_{n}$, Proposition 3.3 leads to the fact that $\mathbf{C}^{\bar{\tau}_{n}}(2 \alpha(n) M+a ; S, \lambda)$ is closed under convergence in probability.

Recall that the set $\left\{\left\|\phi^{1}\right\|_{\bar{\tau}_{n}}:\left(\phi^{0}, \phi^{1}\right) \in \mathcal{A}_{2 \alpha(n) M+a}(\lambda ; S)\right\}$ is bounded in probability and $V^{m}$ converges to $V$, $\mathbb{P}$-a.s. Following an argument used in the first part of the proof Proposition 3.3, which uses the compactness lemma of finite variation processes (see, e.g., lemma B.4 of Guasoni et al., 2012), we can assume (up to choosing a sequence of forward convex combinations) that the sequence $\left(\phi^{0, m}, \phi^{1, m}\right)$ converges pointwise to a predictable and finite variation process $\left(\phi^{0, *}, \phi^{1, *}\right) \in \mathcal{A}_{2 \alpha(n) M+a}$. Because $\mathbf{C}^{\bar{\tau}_{n}}(2 \alpha(n) M+a ; S, \lambda)$ is closed under convergence in probability, it follows that $V_{\bar{\tau}_{n}}^{\text {liq, }}\left(\phi^{0, m}, \phi^{1, m}\right)$ converges $\mathbb{P}$-a.s. to the random variable $V_{\bar{\tau}_{n}}^{\text {liq,0 }}\left(\phi^{0, *}, \phi^{1, *}\right)$. Moreover, as $\left|\phi_{t}^{1, m}\right| \leq M$ for the fixed level $M>0$ and $0 \leq t \leq \bar{\tau}_{n}$, we obtain that $\left|\phi_{t}^{1, *}\right| \leq M$ for $0 \leq t \leq \bar{\tau}_{n}$. Therefore, we can conclude that $V \leq V_{\bar{\tau}_{n}}^{\text {liq,0 }}\left(\phi^{0, *}, \phi^{1, *}\right)$, i.e., $V \in \mathbf{C}_{M}^{\bar{\tau}_{n}}(2 \alpha(n) M+a ; \lambda, S) \subset \mathbf{C}_{M}^{\bar{\tau}_{n}}$, which completes the proof.

Lemma 3.9. If the stock price process $S$ with the transaction cost $\lambda$ satisfies the NUPBR and NLABP conditions in the robust sense with the smaller bid-ask spread pair $\left(S^{\prime}, \lambda^{\prime}\right)$, there exists another bidask spread pair $(\breve{S}, \breve{\lambda})$ satisfying Assumption 2.1 , which stays strictly between the two spreads in the sense that

$$
\begin{aligned}
\inf _{t \in[0, T]}\left(\left(1+\breve{\lambda}_{t}\right) \breve{S}_{t}-\left(1+\lambda_{t}^{\prime}\right) S_{t}^{\prime}\right)>0, & \inf _{t \in[0, T]}\left(\left(1+\lambda_{t}\right) S_{t}-\left(1+\breve{\lambda}_{t}\right) \breve{S}_{t}\right)>0, \\
\text { and } \inf _{t \in[0, T]}\left(\left(1-\lambda_{t}^{\prime}\right) S_{t}^{\prime}-\left(1-\breve{\lambda}_{t}\right) \breve{S}_{t}\right)>0, & \inf _{t \in[0, T]}\left(\left(1-\breve{\lambda}_{t}\right) \breve{S}_{t}-\left(1-\lambda_{t}\right) S_{t}\right)>0 \text {, a.s. }
\end{aligned}
$$

Moreover, the stock price process $\left(\breve{S}_{t}\right)_{t \in[0, T]}$ satisfies the RNUPBR and NLABP conditions with the transaction costs $\left(\breve{\lambda}_{t}\right)_{t \in[0, T]}$.

Proof. Assume that the pair $(S, \lambda)$ satisfies the NUPBR and NLABP conditions with the smaller spread pair $\left(S^{\prime}, \lambda^{\prime}\right)$. Let us define the auxiliary pair of processes

$$
\breve{S}_{t} \triangleq \frac{S_{t}+S_{t}^{\prime}}{2}, \quad \breve{\lambda}_{t} \triangleq \frac{\lambda_{t} S_{t}+\lambda_{t}^{\prime} S_{t}^{\prime}}{S_{t}+S_{t}^{\prime}} \in(0,1), \quad \forall t \in[0, T]
$$

It is clear that the new pair $(\breve{S}, \breve{\lambda})$ satisfies Assumption 2.1. We claim that the stock price process $\breve{S}$ with transaction costs $\breve{\lambda}$ satisfies the inequalities (3.15). Indeed, it is enough to notice that

$$
\begin{aligned}
\inf _{t \in[0, T]}\left(\left(1+\breve{\lambda}_{t}\right) \breve{S}_{t}-\left(1+\lambda_{t}^{\prime}\right) S_{t}^{\prime}\right) & =\inf _{t \in[0, T]}\left(\left(1+\lambda_{t}\right) S_{t}-\left(1+\breve{\lambda}_{t}\right) \breve{S}_{t}\right) \\
& =\inf _{t \in[0, T]}\left(\frac{1}{2}\left(1+\lambda_{t}\right) S_{t}-\frac{1}{2}\left(1+\lambda_{t}^{\prime}\right) S_{t}^{\prime}\right)>0 \text {, a.s. }
\end{aligned}
$$


Similarly, we also have

$$
\begin{aligned}
\inf _{t \in[0, T]}\left(\left(1-\lambda_{t}^{\prime}\right) S_{t}^{\prime}-\left(1-\breve{\lambda}_{t}\right) \breve{S}_{t}\right) & =\inf _{t \in[0, T]}\left(\left(1-\breve{\lambda}_{t}\right) \breve{S}_{t}-\left(1-\lambda_{t}\right) S_{t}\right) \\
& =\inf _{t \in[0, T]}\left(\frac{1}{2}\left(1-\lambda_{t}^{\prime}\right) S_{t}^{\prime}-\frac{1}{2}\left(1-\lambda_{t}\right) S_{t}\right)>0 \text {, a.s. }
\end{aligned}
$$

Therefore, the inequalities in (3.15) are verified.

Recall that the stock price process $S^{\prime}$ with the transaction cost $\lambda^{\prime}$ satisfies the NUPBR and NLABP conditions. We aim to verify that the stock price process $\breve{S}$ with the transaction cost $\breve{\lambda}$ satisfies the RNUPBR and NLABP conditions.

First, it is trivial to see that $\breve{S}$ satisfies RNUPBR as the pair $\left(S^{\prime}, \lambda^{\prime}\right)$ satisfies the NUPBR condition. On the other hand, if $\left(\phi^{0}, \phi^{1}\right)$ is self-financing for the pair $(\breve{S}, \breve{\lambda})$ such that (2.1) is satisfied, then $\left(\phi^{0}, \phi^{1}\right)$ is also self-financing for the pair $\left(S^{\prime}, \lambda^{\prime}\right)$ because $\left(1-\breve{\lambda}_{t}\right) \breve{S}_{t}<\left(1-\lambda_{t}^{\prime}\right) S_{t}$ and $\left(1+\breve{\lambda}_{t}\right) \breve{S}_{t}>\left(1+\lambda_{t}^{\prime}\right) S_{t}$ a.s., $t \in[0, T]$. In addition, we also get that $V_{t}^{\text {liq, } x ; \breve{S}, \breve{\lambda}}\left(\phi^{0}, \phi^{1}\right)<V_{t}^{\text {liq }, x ; S^{\prime}, \lambda^{\prime}}\left(\phi^{0}, \phi^{1}\right)$ a.s., $t \in[0, T]$, for the same self-financing portfolio $\left(\phi^{0}, \phi^{1}\right)$. It thereby follows that $\mathcal{A}_{x}(\breve{\lambda} ; \breve{S}) \subset \mathcal{A}_{x}\left(\lambda^{\prime} ; S^{\prime}\right)$. If there exists an arbitrage opportunity with a stopping time $\tau$ where $\mathbb{P}(\tau<T)>0$ and a bounded portfolio $\left(\phi^{0}, \phi^{1}\right) \in$ $\mathcal{A}_{x}^{\text {bd }}(\breve{\lambda} ; \breve{S})$, it is obvious that $\left(\phi^{0}, \phi^{1}\right)$ also leads to an LA opportunity for the pair $\left(S^{\prime}, \lambda^{\prime}\right)$. In other words, we can deduce that if the stock price process $S^{\prime}$ satisfies the NLABP condition with the transaction cost $\lambda^{\prime}$, then the stock price process $\breve{S}$ also satisfies the NLABP condition with the transaction cost $\breve{\lambda}$.

The following result is the last important preparation for the proof of the implication $(1) \Rightarrow(2)$ in Theorem 2.8; see lemma 6.3 and its proof in Guasoni et al. (2012).

Lemma 3.10. Let $\left(X_{t}\right)_{t \in[0, T]}$ and $\left(Y_{t}\right)_{t \in[0, T]}$ be two càdlàg bounded processes. The following conditions are equivalent:

(i) There exists a càdlàg martingale $\left(M_{t}\right)_{t \in[0, T]}$ such that

$$
X \leq M \leq Y, \quad \text { a.s. }
$$

(ii) For all stopping times $\sigma$, $\tau$ such that $0 \leq \sigma \leq \tau \leq T$ a.s., we have

$$
\mathbb{E}\left[X_{\tau} \mid \mathcal{F}_{\sigma}\right] \leq Y_{\sigma} \text { and } \mathbb{E}\left[Y_{\tau} \mid \mathcal{F}_{\sigma}\right] \geq X_{\sigma} \text { a.s. }
$$

We now proceed to finish the proof of Theorem 2.8.

Proof of $(\mathbf{1}) \Rightarrow$ (2) of Theorem 2.8. If the stock price process $S$ satisfies the NUPBR and NLABP conditions in the robust sense with the transaction cost $\lambda$, Lemma 3.9 guarantees the existence of the auxiliary pair $(\breve{S}, \breve{\lambda})$ such that $\breve{S}$ satisfies the RNUPBR condition and NLABP condition with the transaction cost $\breve{\lambda}$.

In the next few steps, let us consider the market model with the stock price process $\breve{S}$ and the transaction cost $\breve{\lambda}$. If we replace the pair $(S, \lambda)$ by the auxiliary pair $(\breve{S}, \breve{\lambda})$, all conclusions from Lemma 3.2 to Lemma 3.8 still hold for $(\breve{S}, \breve{\lambda})$. For some fixed large level $M>0$, consider the $\operatorname{set} \mathbf{C}_{M}^{\bar{\tau}_{n}}(\breve{S}, \breve{\lambda})$. Combining the facts that $\mathbf{C}_{M}^{\bar{\tau}_{n}}(\breve{S}, \breve{\lambda}) \cap \mathbb{L}^{\infty}$ is Fatou closed (due to Lemma 3.8) and $\mathbf{C}_{M}^{\bar{\tau}_{n}}(\breve{S}, \breve{\lambda}) \cap \mathbb{L}_{+}^{\infty}=\{0\}$ (due to Lemma 3.6) where the stopping times $\left\{\bar{\tau}_{n}\right\}_{n \in \mathbb{N}}$ are defined in (3.14), there exists a probability measure $\mathbb{Q}^{n}$ equivalent to $\mathbb{P}$ such that for any $V \in \mathbf{C}_{M}^{\bar{\tau}_{n}}(\breve{S}, \breve{\lambda}) \cap \mathbb{L}^{\infty}$, we have $\mathbb{E}^{\mathbb{Q}^{n}}[V] \leq 0$, where we used lemma 5.5.2 of Kabanov and Safarian (2009) (which relates Fatou-closedness to weak-star closedness) and the Kreps-Yan separation theorem (see, e.g., theorem B.3 of Guasoni et al., 2012). 
In particular, for each $n \in \mathbb{N}$, let us only consider the subset $\overline{\mathbf{C}}_{M}^{\bar{\tau}_{n}}(\breve{S}, \breve{\lambda})$ such that the portfolio process $\phi^{1}$ is a nonpositive process or a nonnegative process on $\llbracket 0, \bar{\tau}_{n} \rrbracket$ and $\phi_{\bar{\tau}_{n}}^{1}=0$; i.e., the position in the stock at the terminal time $\bar{\tau}_{n}$ will be liquidated,

$$
\begin{gathered}
\overline{\mathbf{C}}_{M}^{\bar{\tau}_{n}}(\breve{S}, \breve{\lambda}) \triangleq\left\{V: V \leq V_{\bar{\tau}_{n}}^{\text {liq, }, 0}\left(\phi^{0}, \phi^{1}\right) \in \mathcal{A}_{x} \text { where } \phi^{1}\right. \text { is a nonpositive or a nonnegative process } \\
\left.\phi_{\bar{\tau}_{n}}^{1}=0, \text { and }\left|\phi_{t}^{1}\right| \leq M, \mathbb{P} \text {-a.s. on } \llbracket 0, \bar{\tau}_{n} \rrbracket\right\}
\end{gathered}
$$

with $M$ large enough for which we have $\mathbb{E}^{\mathbb{Q}^{n}}[V] \leq 0$ for all $V \in \overline{\mathbf{C}}_{M}^{\bar{\tau}_{n}}(\breve{S}, \breve{\lambda}) \cap \mathbb{L}^{\infty} \subset \mathbf{C}_{M}^{\bar{\tau}_{n}}(\breve{S}, \breve{\lambda}) \cap \mathbb{L}^{\infty}$.

Let us recall that $\breve{S}_{t} \leq 2 \alpha(n)$ for $t \leq \bar{\tau}_{n}$ as the original stock price process $S_{t} \leq \alpha(n)$ for $t \leq \bar{\tau}_{n}$. We consider the following portfolio in the market driven by $\breve{S}$ with transaction costs $\breve{\lambda}$,

$$
\begin{aligned}
& \tilde{\phi}^{0} \triangleq\left[\left(1-\breve{\lambda}_{\eta}\right) \breve{S}_{\eta} \mathbf{1}_{\rrbracket \eta, \sigma \llbracket}(t)+\left(\left(1-\breve{\lambda}_{\eta}\right) \breve{S}_{\eta}-\left(1+\breve{\lambda}_{\sigma}\right) \breve{S}_{\sigma}\right) \mathbf{1}_{\llbracket \sigma, \bar{\tau}_{n} \rrbracket}\right] \mathbf{1}_{A}, \\
& \tilde{\phi}^{1} \triangleq-\mathbf{1}_{\rrbracket \eta, \sigma \llbracket}(t) \mathbf{1}_{A},
\end{aligned}
$$

for any stopping time $\eta \leq \sigma \leq \bar{\tau}_{n}$ and $A \in \mathcal{F}_{\eta}$. Similar to the proof of Lemma 3.5, it is not hard to check that $\left(\tilde{\phi}^{0}, \tilde{\phi}^{1}\right)$ is self-financing.

Similarly, we consider the portfolio

$$
\begin{aligned}
& \hat{\phi}^{0} \triangleq\left[-\left(1+\breve{\lambda}_{\eta}\right) \breve{S}_{\eta} \mathbf{1}_{\rrbracket \eta, \sigma \llbracket}(t)+\left(-\left(1+\breve{\lambda}_{\eta}\right) \breve{S}_{\eta}+\left(1-\breve{\lambda}_{\sigma}\right) \breve{S}_{\sigma}\right) \mathbf{1}_{\llbracket \sigma, \bar{\tau}_{n} \rrbracket}\right] \mathbf{1}_{A}, \\
& \hat{\phi}^{1} \triangleq \mathbf{1}_{\rrbracket \eta, \sigma \llbracket}(t) \mathbf{1}_{A},
\end{aligned}
$$

for any stopping time $\eta \leq \sigma \leq \bar{\tau}_{n}$ and $A \in \mathcal{F}_{\eta}$. We can similarly verify that $\left(\hat{\phi}^{0}, \hat{\phi}^{1}\right)$ is also selffinancing.

It is also easy to show that $V_{\bar{\tau}_{n}}^{\text {liq,0 }}\left(\tilde{\phi}^{0}, \tilde{\phi}^{1}\right) \in \overline{\mathbf{C}}_{M}^{\bar{\tau}_{n}}(\breve{S}, \breve{\lambda}) \cap \mathbb{L}^{\infty}$ and $V_{\bar{\tau}_{n}}^{\text {liq,0 }}\left(\hat{\phi}^{0}, \hat{\phi}^{1}\right) \in \overline{\mathbf{C}}_{M}^{\bar{\tau}_{n}}(\breve{S}, \breve{\lambda}) \cap \mathbb{L}^{\infty}$ as $\tilde{\phi}^{1}$ is nonpositive, $\hat{\phi}$ is nonnegative, and $|\tilde{\phi}|,|\hat{\phi}|$, and $\breve{S}$ are all uniformly bounded on $\llbracket 0, \bar{\tau}_{n} \rrbracket$. In particular, we have

$$
\begin{aligned}
V_{\bar{\tau}_{n}}^{\mathrm{liq}, 0}\left(\tilde{\phi}^{0}, \tilde{\phi}^{1}\right) & =\tilde{\phi}_{\bar{\tau}_{n}}^{0}+\left(\tilde{\phi}_{\bar{\tau}_{n}}^{1}\right)^{+}\left(1-\breve{\lambda}_{\bar{\tau}_{n}}\right) \breve{S}_{\bar{\tau}_{n}}-\left(\tilde{\phi}_{\bar{\tau}_{n}}^{1}\right)^{-}\left(1+\breve{\lambda}_{\bar{\tau}_{n}}\right) \breve{S}_{\bar{\tau}_{n}} \\
& =\left(\left(1-\breve{\lambda}_{\eta}\right) \breve{S}_{\eta}-\left(1+\breve{\lambda}_{\sigma}\right) \breve{S}_{\sigma}\right) \mathbf{1}_{A}, \\
V_{\bar{\tau}_{n}}^{\text {liq, } 0}\left(\hat{\phi}^{0}, \hat{\phi}^{1}\right) & =\hat{\phi}_{\bar{\tau}_{n}}^{0}+\left(\hat{\phi}_{\bar{\tau}_{n}}^{1}\right)^{+}\left(1-\breve{\lambda}_{\bar{\tau}_{n}}\right) \breve{S}_{\bar{\tau}_{n}}-\left(\hat{\phi}_{\bar{\tau}_{n}}^{1}\right)^{-}\left(1+\breve{\lambda}_{\bar{\tau}_{n}}\right) \breve{S}_{\bar{\tau}_{n}} \\
& =\left(-\left(1+\breve{\lambda}_{\eta}\right) \breve{S}_{\eta}+\left(1-\breve{\lambda}_{\sigma}\right) \breve{S}_{\sigma}\right) \mathbf{1}_{A} .
\end{aligned}
$$

By the inequalities $\mathbb{E}^{\mathbb{Q}^{n}}\left[V_{\bar{\tau}_{n}}^{\text {liq,0 }}\left(\tilde{\phi}^{0}, \tilde{\phi}^{1}\right)\right] \leq 0$ and $\mathbb{E}^{\mathbb{Q}^{n}}\left[V_{\bar{\tau}_{n}}^{\text {liq, } 0}\left(\hat{\phi}^{0}, \hat{\phi}^{1}\right)\right] \leq 0$, it is easy to obtain that

$$
\begin{aligned}
& \mathbb{E}^{\mathbb{Q}^{n}}\left[\breve{S}_{\sigma}\left(1+\breve{\lambda}_{\sigma}\right) \mid \mathcal{F}_{\eta}\right] \geq \breve{S}_{\eta}\left(1-\breve{\lambda}_{\eta}\right), \\
& \mathbb{E}^{\mathbb{Q}^{n}}\left[\breve{S}_{\sigma}\left(1-\breve{\lambda}_{\sigma}\right) \mid \mathcal{F}_{\eta}\right] \leq \breve{S}_{\eta}\left(1+\breve{\lambda}_{\eta}\right)
\end{aligned}
$$

Lemma 3.10 implies the existence of a càdlàg martingale $\tilde{S}^{n}$ under $\mathbb{Q}^{n}$ such that

$$
\breve{S}_{t}\left(1-\breve{\lambda}_{t}\right) \leq \tilde{S}_{t}^{n} \leq \breve{S}_{t}\left(1+\breve{\lambda}_{t}\right), \text { a.s. for } 0 \leq t \leq \bar{\tau}_{n} .
$$


So far, for each $n \in \mathbb{N}$, we obtain the pair $\left(\mathbb{Q}^{n}, \tilde{S}^{n}\right)$ on $\llbracket 0, \bar{\tau}_{n} \rrbracket$ such that $\tilde{S}^{n}$ is a martingale under $\mathbb{Q}^{n}$ and $\tilde{S}^{n}$ evolves inside the spread $[(1-\breve{\lambda}) \breve{S},(1+\breve{\lambda}) \breve{S}]$. However, in general, we may not have the concatenation property of two measures $\mathbb{Q}^{n}$ and $\mathbb{Q}^{n-1}$ such that $\left.\mathbb{Q}^{n}\right|_{\mathcal{F}_{\bar{\tau}_{n-1}}}=\left.\mathbb{Q}^{n-1}\right|_{\mathcal{F}_{\bar{\tau}_{n-1}}}$. Therefore, we cannot simply paste the processes $\left\{\tilde{S}^{n}\right\}_{n \in \mathbb{N}}$ together over the whole interval $[0, T]$ to obtain the desired process $\tilde{S}$. To finish the proof, we need several further steps using the elements $\left(\mathbb{Q}^{n}, \tilde{S}^{n}\right)$ that we already have.

For each $\mathbb{Q}^{n}$, let us consider the density process and the corresponding stochastic exponential

$$
\begin{aligned}
Z_{t}^{n} & \triangleq \mathbb{E}\left[\frac{d \mathbb{Q}^{n}}{d \mathbb{P}} \mid \mathcal{F}_{t}\right], \quad t \in[0, T], \\
\mathcal{E}_{t}\left(Y^{n}\right) & \triangleq Z_{t \wedge \bar{\tau}_{n}}^{n}, \quad t \in[0, T],
\end{aligned}
$$

where $Y^{n}$ is a local martingale.

Similar to the proof of lemma 3.5 of Choulli et al. (2015), we define the stochastic process using stochastic integrals

$$
Y \triangleq \sum_{n=1}^{\infty} \mathbf{1}_{\| \bar{\tau}_{n-1}, \bar{\tau}_{n} \rrbracket} \cdot Y^{n} .
$$

It is easy to see that $\left(Y_{t}\right)_{t \in[0, T]}$ is a local martingale with initial value $Y_{0}=0$ as

$$
Y_{t \wedge \bar{\tau}_{n}}=\left(\sum_{k=1}^{n} \mathbf{1}_{\rrbracket \bar{\tau}_{k-1}, \bar{\tau}_{k} \rrbracket} \cdot Y^{k}\right)_{t}
$$

is a true martingale. As a consequence, the stochastic exponential $\mathcal{E}(Y)$ is a local martingale. Denote $\left\{v_{n}\right\}_{n \in \mathbb{N}}$ the localizing sequence of the local martingale $\mathcal{E}(Y)$, we shall consider the new localizing sequence $\left\{v_{n} \wedge \bar{\tau}_{n}\right\}_{n \in \mathbb{N}}$ that converges to $T$. To simplify the notation, let us still denote this sequence of stopping times by $\left\{\bar{\tau}_{n}\right\}_{n \in \mathbb{N}}$.

Notice that $\mathcal{E}\left(Y^{n}\right)>0$ for all $n \in \mathbb{N}$ as it is the density process of $\frac{d \mathbb{Q}^{n}}{d \mathbb{P}}$. It follows that $\mathcal{E}(Y)>0$ because $1+\triangle Y=1+\triangle Y^{n}>0$ on $\rrbracket \bar{\tau}_{k-1}, \bar{\tau}_{k} \rrbracket, n \in \mathbb{N}$.

Let us focus on the positive local martingale

$$
Z \triangleq \mathcal{E}(Y)
$$

and consider the sequence of probability measures induced by

$$
\frac{d \hat{\mathbb{Q}}^{n}}{d \mathbb{P}} \triangleq Z_{\bar{\tau}_{n}}=\mathcal{E}(Y)_{\bar{\tau}_{n}}=\mathcal{E}\left(Y_{\bar{\tau}_{n}}\right)
$$

Clearly, the sequence of probability measures $\left\{\hat{\mathbb{Q}}^{n}\right\}_{n \in \mathbb{N}}$ satisfies the desired concatenation property. We now claim that for any $V \in \overline{\mathbf{C}}_{M}^{\bar{\tau}_{n}}(\breve{S}, \breve{\lambda}) \cap \mathbb{L}^{\infty}$, the inequality $\mathbb{E}^{\hat{\mathbb{Q}}^{n}}[V] \leq 0$ still holds. To prove the claim, we recall the existence of $\left(\phi^{0}, \phi^{1}\right) \in \mathcal{A}_{x}$ for some $x \geq 0$ where $\phi^{1}$ is either a nonpositive process or a nonnegative process with $\phi_{\bar{\tau}_{1}}=0$ and $\left|\phi^{1}\right| \leq M, \mathbb{P}$-a.s. on $\llbracket 0, \bar{\tau}_{n} \rrbracket$ such that $V \leq V_{\bar{\tau}_{n}}^{\text {liq, } 0}\left(\phi^{0}, \phi^{1}\right)$.

Case 1: The portfolio process $\phi^{1}$ is a nonpositive process on $\llbracket 0, \bar{\tau}_{n} \rrbracket$.

For each fixed choice of $n \in \mathbb{N}$, we first consider the fictitious stock price processes constructed inductively for $1 \leq k \leq n$. First, for $0 \leq t \leq \bar{\tau}_{1}$, let us define the stock process

$$
\bar{S}_{t}^{1} \triangleq \tilde{S}_{t}^{1}
$$

which is a martingale under $\mathbb{Q}^{1}$ and stays in the spread $[(1-\breve{\lambda}) \breve{S},(1+\breve{\lambda}) \breve{S}]$. 
Next, for all $\bar{\tau}_{1} \leq t \leq \bar{\tau}_{2}$, we consider two auxiliary processes

$$
\begin{aligned}
& X_{t}^{\prime} \triangleq \underset{\tau \in \mathcal{O}_{t}}{\operatorname{ess} \sup } \mathbb{E}^{\mathbb{Q}^{2}}\left[\breve{S}_{\tau}\left(1+\frac{\bar{S}_{\bar{\tau}_{1}}^{1}-\breve{S}_{\bar{\tau}_{1}}}{\breve{S}_{\bar{\tau}_{1}} \breve{\lambda}_{\bar{\tau}_{1}}} \breve{\lambda}_{\tau}\right) \mid \mathcal{F}_{t}\right] \\
& Y_{t}^{\prime} \triangleq \underset{\tau \in \mathcal{O}_{t}}{\operatorname{essinf}} \mathbb{E}^{\mathbb{Q}^{2}}\left[\breve{S}_{\tau}\left(1+\breve{\lambda}_{\tau}\right) \mid \mathcal{F}_{t}\right] \text {, }
\end{aligned}
$$

where $\mathcal{O}_{t}$ denotes the set of all stopping times $\tau$ with values such that $t \leq \tau \leq \bar{\tau}_{2}$. For $0 \leq t \leq \bar{\tau}_{1}$, we shall define the processes

$$
X_{t} \triangleq \mathbb{E}^{\mathbb{Q}^{2}}\left[X_{\bar{\tau}_{1}}^{\prime} \mid \mathcal{F}_{t}\right], \quad Y_{t} \triangleq \mathbb{E}^{\mathbb{Q}^{2}}\left[Y_{\bar{\tau}_{1}}^{\prime} \mid \mathcal{F}_{t}\right]
$$

Next, for $\bar{\tau}_{1} \leq t \leq \bar{\tau}_{2}$, we define $X_{t} \triangleq X_{t}^{\prime}$ and $Y_{t} \triangleq Y_{t}^{\prime}$. Similar to the proof of lemma 6.3 of Guasoni et al. (2012), we get that $(X)_{t \wedge \bar{\tau}_{2}}$ is a supermartingale and $(Y)_{t \wedge \bar{\tau}_{2}}$ is a submartingale under $\mathbb{Q}^{2}$. In addition, the fact that $\left(1-\breve{\lambda}_{\bar{\tau}_{1}}\right) \breve{S}_{\bar{\tau}_{1}} \leq \bar{S}_{\bar{\tau}_{1}}^{1}=\tilde{S}_{\bar{\tau}_{1}}^{1} \leq\left(1+\breve{\lambda}_{\bar{\tau}_{1}}\right) \breve{S}_{\bar{\tau}_{1}}$ implies that

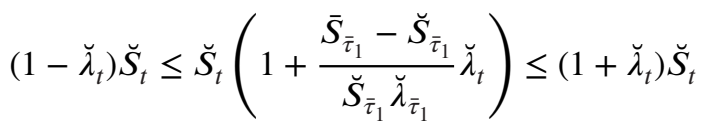

for all $\bar{\tau}_{1} \leq t \leq \bar{\tau}_{2}$. Therefore, by lemma 6.2 of Guasoni et al. (2012), there exists a martingale $M^{2}$ under $\mathbb{Q}^{2}$ such that $X_{t} \leq M_{t}^{2} \leq Y_{t}$ for $0 \leq t \leq \bar{\tau}_{2}$. In particular, we have that $\left(1-\breve{\lambda}_{t}\right) \breve{S}_{t} \leq M_{t}^{2} \leq\left(1+\breve{\lambda}_{t}\right) \breve{S}_{t}$ for $\bar{\tau}_{1} \leq t \leq \bar{\tau}_{2}$ and also

$$
M_{\bar{\tau}_{1}}^{2} \geq \breve{S}_{\bar{\tau}_{1}}\left(1+\frac{\bar{S}_{\bar{\tau}_{1}}-\breve{S}_{\bar{\tau}_{1}}}{\breve{S}_{\bar{\tau}_{1}} \breve{\lambda}_{\bar{\tau}_{1}}}\right)=\bar{\lambda}_{\bar{\tau}_{1}}^{1}, \mathbb{Q}^{2} \text {-a.s. }
$$

We now consider the auxiliary stock price process for $0 \leq t \leq \bar{\tau}_{2}$ defined by

$$
\bar{S}_{t}^{2} \triangleq\left\{\begin{aligned}
\tilde{S}_{t}^{2}, & \text { for } 0 \leq t<\bar{\tau}_{1}, \\
\max \left(\tilde{S}_{t}^{2}, M_{t}^{2}\right), & \text { for } \bar{\tau}_{1} \leq t \leq \bar{\tau}_{2} .
\end{aligned}\right.
$$

It is easy to show that $\left(\bar{S}^{2}\right)_{t \wedge \bar{\tau}_{2}}$ is a submartingale under $\mathbb{Q}^{2}$ as both $\tilde{S}^{2}$ and $M^{2}$ are martingales under $\mathbb{Q}^{2}$ for $0 \leq t \leq \bar{\tau}_{2}$. Moreover, by its construction, we obtain that $\bar{S}^{2}$ stays in the spread, i.e., $\left(1-\breve{\lambda}_{t}\right) \breve{S}_{t} \leq$ $\bar{S}_{t}^{2} \leq\left(1+\breve{\lambda}_{t}\right) \breve{S}_{t}$ for $0 \leq t \leq \bar{\tau}_{2}$ as well as the ordering $\bar{S}_{\bar{\tau}_{1}}^{2} \geq \bar{S}_{\bar{\tau}_{1}}^{1} \mathbb{Q}^{2}$-a.s.

By repeating this construction, for $2 \leq k \leq n$, we can first get the existence of a martingale $M^{k}$ under $\mathbb{Q}^{k}$ for $0 \leq t \leq \bar{\tau}_{k}$ that satisfies $\left(1-\breve{\lambda}_{t}\right) \breve{S}_{t} \leq M_{t}^{k} \leq\left(1+\breve{\lambda}_{t}\right) \breve{S}_{t}$ for $\bar{\tau}_{k-1} \leq t \leq \bar{\tau}_{k}$ and $M_{\bar{\tau}_{k-1}}^{k} \geq \bar{S}_{\bar{\tau}_{k-1}}^{k-1}$ $\mathbb{Q}^{k}$-a.s. Let us then define

$$
\bar{S}_{t}^{k} \triangleq\left\{\begin{aligned}
\tilde{S}_{t}^{k}, & \text { for } 0 \leq t<\bar{\tau}_{k-1}, \\
\max \left(\tilde{S}_{t}^{k}, M_{t}^{k}\right), & \text { for } \bar{\tau}_{k-1} \leq t \leq \bar{\tau}_{k} .
\end{aligned}\right.
$$

We obtain a sequence of processes $\left\{\bar{S}^{k}\right\}_{1 \leq k \leq n}$ such that $\bar{S}_{t}^{k}$ is a submartingale under $\mathbb{Q}^{k}$ and satisfies the spread constraint $\left(1-\breve{\lambda}_{t}\right) \breve{S}_{t} \leq \bar{S}_{t}^{k} \leq\left(1+\breve{\lambda}_{t}\right) \breve{S}_{t}$ for $0 \leq t \leq \bar{\tau}_{k}$, and at each stopping time, we have the inequality

$$
\bar{S}_{\bar{\tau}_{k-1}}^{k} \geq \bar{S}_{\bar{\tau}_{k-1}}^{k-1}, \mathbb{Q}^{k} \text {-a.s. }
$$


For the future purpose, we complete the definition of each auxiliary process $\bar{S}_{t}^{k}$ after $t \geq \bar{\tau}_{k}$ by setting

$$
\bar{S}_{t}^{k}=\bar{S}_{\bar{\tau}_{k}}^{k}, \quad t \geq \bar{\tau}_{k}, \text { for } k=1, \ldots, n .
$$

Let us consider the fictitious semimartingale stock price process defined by

$$
S_{t}^{\diamond} \triangleq \begin{cases}\bar{S}_{t}^{k}, & \text { for } \bar{\tau}_{k-1} \leq t<\bar{\tau}_{k}, \quad k=1, \ldots, n \\ \bar{S}_{\bar{\tau}_{n}}^{n}, & \text { for } t \geq \bar{\tau}_{n} .\end{cases}
$$

It is clear that $\left(1-\lambda_{t}\right) S_{t} \leq S_{t}^{\diamond} \leq\left(1+\lambda_{t}\right) S_{t}$ for $0 \leq t \leq \bar{\tau}_{n}$. Similar to the proof of Proposition 3.1, we can deduce that

$$
V_{t \wedge \bar{\tau}_{n}}^{\mathrm{liq}, 0}\left(\phi^{0}, \phi^{1}\right) \leq\left(\phi^{1} \cdot S^{\diamond}\right)_{t \wedge \bar{\tau}_{n}}=\sum_{k=1}^{n}\left(\phi^{1} \cdot\left(\mathbf{1}_{\llbracket \bar{\tau}_{k-1}, \bar{\tau}_{k} \llbracket} \bar{S}^{k}\right)\right)_{t \wedge \bar{\tau}_{n}} .
$$

We aim to prove that

$$
\mathbb{E}^{\hat{\mathbb{Q}}^{n}}\left[\sum_{k=1}^{n}\left(\phi^{1} \cdot\left(\mathbf{1}_{\llbracket \bar{\tau}_{k-1}, \bar{\tau}_{k} \llbracket} \bar{S}^{k}\right)\right)_{\bar{\tau}_{n}}\right]=\sum_{k=1}^{n} \mathbb{E}\left[\mathcal{E}\left(Y_{\bar{\tau}_{n}}\right)\left(\phi^{1} \cdot\left(\mathbf{1}_{\llbracket \bar{\tau}_{k-1}, \bar{\tau}_{k} \llbracket} \bar{S}^{k}\right)\right)_{\bar{\tau}_{n}}\right] \leq 0 .
$$

For $1 \leq k \leq n$, using the integration by parts, we can deduce that

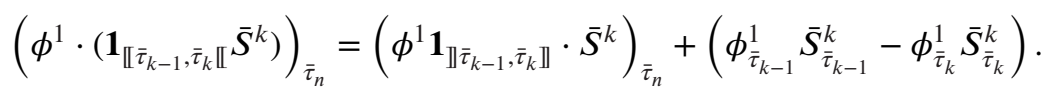

Consequently, proving (3.21) is equivalent to showing that the following holds:

$$
\mathbb{E}\left[\mathcal{E}\left(Y_{\bar{\tau}_{n}}\right) \sum_{k=1}^{n}\left(\phi^{1} \mathbf{1}_{\rrbracket \bar{\tau}_{k-1}, \bar{\tau}_{k} \rrbracket} \cdot \bar{S}^{k}\right)_{\bar{\tau}_{n}}+\mathcal{E}\left(Y_{\bar{\tau}_{n}}\right) \sum_{k=1}^{n}\left(\phi_{\bar{\tau}_{k-1}}^{1} \bar{S}_{\bar{\tau}_{k-1}}^{k}-\phi_{\bar{\tau}_{k}}^{1} \bar{S}_{\bar{\tau}_{k}}^{k}\right)\right] \leq 0 .
$$

For the first part in (3.22), we claim that $\mathcal{E}(Y)_{t \wedge \bar{\tau}_{n}}\left(\phi^{1} \mathbf{1}_{\rrbracket \bar{\tau}_{k-1}, \bar{\tau}_{k} \rrbracket} \cdot \bar{S}^{k}\right)_{t \wedge \bar{\tau}_{n}}$ is a local supermartingale under $\mathbb{P}$. Similar to the proof of lemma 3.5 of Choulli et al. (2015), Itô's lemma yields that it is equivalent to prove that

$$
\left(\mathbf{1}_{\rrbracket \bar{\tau}_{k-1}, \bar{\tau}_{k} \rrbracket} \phi^{1} \cdot \bar{S}^{k}+\mathbf{1}_{\rrbracket \bar{\tau}_{k-1}, \bar{\tau}_{k} \rrbracket} \phi^{1} \cdot\left[\bar{S}^{k}, Y\right]\right)_{t \wedge \bar{\tau}_{n}}
$$

is a local supermartingale under $\mathbb{P}$ with initial value 0 . However, it is clear by the definition of $Y$ that

$$
\left(\mathbf{1}_{\rrbracket \bar{\tau}_{k-1}, \bar{\tau}_{k} \rrbracket} \phi^{1} \cdot \bar{S}^{k}+\mathbf{1}_{\rrbracket \bar{\tau}_{k-1}, \bar{\tau}_{k} \rrbracket} \phi^{1} \cdot\left[\bar{S}^{k}, Y\right]\right)_{t}=\left(\mathbf{1}_{\rrbracket \bar{\tau}_{k-1}, \bar{\tau}_{k} \rrbracket} \phi^{1} \cdot \bar{S}^{k}+\mathbf{1}_{\rrbracket \bar{\tau}_{k-1}, \bar{\tau}_{k} \rrbracket} \phi^{1} \cdot\left[\bar{S}^{k}, Y^{k}\right]\right)_{t} .
$$

Notice that $\bar{S}^{k}$ is a submartingale under $\mathbb{Q}^{k}$. Therefore, $\mathcal{E}\left(Y^{k}\right)_{t \wedge \bar{\tau}_{n}} \bar{S}_{t \wedge \bar{\tau}_{n}}^{k}$ is a submartingale under $\mathbb{P}$. We claim that $\left(\bar{S}^{k}+\left[\bar{S}^{k}, Y^{k}\right]\right)_{t \wedge \bar{\tau}_{n}}$ is also a submartingale under $\mathbb{P}$. To prove the claim, we can use the product rule and obtain that

$$
d\left(\mathcal{E}\left(Y^{k}\right)_{t} \bar{S}_{t}^{k}\right)=\bar{S}^{k} d \mathcal{E}\left(Y^{k}\right)_{t}+\mathcal{E}\left(Y^{k}\right) d\left(\bar{S}^{k}+\left[\bar{S}^{k}, Y^{k}\right]\right)_{t} .
$$

The first term on the right-hand side is a true martingale on $\left[0, \bar{\tau}_{n}\right]$ due to the fact that $\left(\bar{S}^{k}\right)_{t \wedge \bar{\tau}_{n}}$ is uniformly bounded. Therefore, we get that $\left(\mathcal{E}\left(Y^{k}\right) \cdot\left(\bar{S}^{k}+\left[\bar{S}^{k}, Y^{k}\right]\right)\right)_{t \wedge \bar{\tau}_{\eta}}$ is a submartingale. Let us consider the semimartingale decomposition of the process $\left(\bar{S}^{k}+\left[\bar{S}^{k}, Y^{k}\right]\right)_{t \wedge \bar{\tau}_{n}}=M_{t}+A_{t}$, where 
$M_{t}$ is a local martingale and $A_{t}$ is a finite variation process. By the submartingale decomposition of $\left(\mathcal{E}\left(Y^{k}\right) \cdot\left(\bar{S}^{k}+\left[\bar{S}^{k}, Y^{k}\right]\right)\right)_{t \wedge \bar{\tau}_{n}}$, we conclude that the finite variation process $\int_{0}^{t \wedge \bar{\tau}_{n}} \mathcal{E}\left(Y^{k}\right)_{u} d A_{u}$ is an increasing finite variation process for $t \leq \bar{\tau}_{n}$. Let us write $A_{t}$ in terms of its Jordan decomposition $A_{t}=A_{t}^{\uparrow}-A_{t}^{\downarrow}$ and $\|A\|_{t}=A_{t}^{\uparrow}+A_{t}^{\downarrow}$. Then, it is easy to see that both $\int_{0}^{t \wedge \bar{\tau}_{n}} \mathcal{E}\left(Y^{k}\right)_{u} d A_{u}^{\uparrow}$ and $\int_{0}^{t \wedge \bar{\tau}_{n}} \mathcal{E}\left(Y^{k}\right)_{u} d A_{u}^{\downarrow}$ are increasing processes and

$$
\left\|\int_{0}^{t \wedge \bar{\tau}_{n}} \mathcal{E}\left(Y^{k}\right)_{u} d A_{u}\right\|=\int_{0}^{t \wedge \bar{\tau}_{n}} \mathcal{E}\left(Y^{k}\right)_{u} d\|A\|_{u}=\int_{0}^{t \wedge \bar{\tau}_{n}} \mathcal{E}\left(Y^{k}\right)_{u} d A_{u}^{\uparrow}+\int_{0}^{t \wedge \bar{\tau}_{n}} \mathcal{E}\left(Y^{k}\right)_{u} d A_{u}^{\downarrow} .
$$

Therefore, the integral $\int_{0}^{t \wedge \bar{\tau}_{n}} \mathcal{E}\left(Y^{k}\right)_{u} d A_{u}$ has the Jordan decomposition

$$
\int_{0}^{t \wedge \bar{\tau}_{n}} \mathcal{E}\left(Y^{k}\right)_{u} d A_{u}=\int_{0}^{t \wedge \bar{\tau}_{n}} \mathcal{E}\left(Y^{k}\right)_{u} d A_{u}^{\uparrow}-\int_{0}^{t \wedge \bar{\tau}_{n}} \mathcal{E}\left(Y^{k}\right)_{u} d A_{u}^{\downarrow} .
$$

On the other hand, $\int_{0}^{t \wedge \bar{\tau}_{n}} \mathcal{E}\left(Y^{k}\right)_{u} d A_{u}$ is an increasing process from the uniqueness (up to a constant difference) of the Jordan decomposition. We therefore have that $\int_{0}^{t \wedge \bar{\tau}_{n}} \mathcal{E}\left(Y^{k}\right)_{u} d A_{u}^{\downarrow}=0$. Because $\mathcal{E}\left(Y^{k}\right)_{t \wedge \bar{\tau}_{n}}>0 \mathbb{P}$-a.s. for all $t$, we obtain that $A_{t}^{\downarrow}=0$ for all $t$. It follows that the finite variation process $A_{t}$ is an increasing process. As a consequence, we get that $\left(\bar{S}^{k}+\left[\bar{S}^{k}, Y^{k}\right]\right)_{t \wedge \bar{\tau}_{n}}=M_{t}+A_{t}$ is a submartingale.

Recalling that $\phi^{1}$ is a nonpositive process, we derive that the stochastic integral

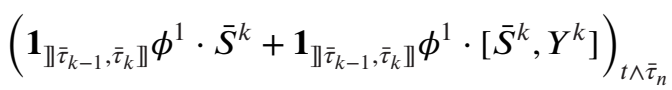

is a local supermartingale under $\mathbb{P}$. It follows that $\mathcal{E}\left(Y_{t \wedge \bar{\tau}_{n}}\right)\left(\mathbf{1}_{\rrbracket \bar{\tau}_{k-1}, \bar{\tau}_{k} \rrbracket} \phi^{1} \cdot \bar{S}^{k}\right)_{t \wedge \bar{\tau}_{n}}$ is a local supermartin-

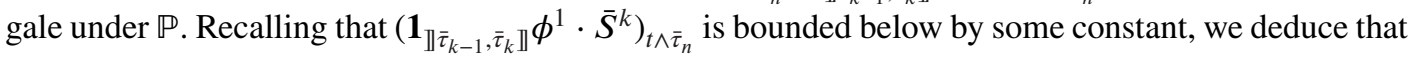
$\mathcal{E}\left(Y_{t \wedge \bar{\tau}_{n}}\right)\left(\mathbf{1}_{\rrbracket \bar{\tau}_{k-1}, \bar{\tau}_{k} \rrbracket} \phi^{1} \cdot \bar{S}^{k}\right)_{t \wedge \bar{\tau}_{n}}$ is bounded below by the martingale $\mathcal{E}\left(Y_{t \wedge \bar{\tau}_{n}}\right)$. Fatou's lemma yields that

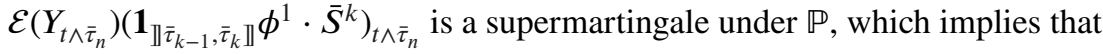

$$
\mathbb{E}\left[\mathcal{E}\left(Y_{\bar{\tau}_{n}}\right) \sum_{k=1}^{n}\left(\phi^{1} \mathbf{1}_{\rrbracket \bar{\tau}_{k-1}, \bar{\tau}_{k} \rrbracket} \cdot \bar{S}^{k}\right)_{\bar{\tau}_{n}}\right] \leq 0 .
$$

For the second part in (3.22), we can rearrange the summation and write

$$
\sum_{k=1}^{n}\left(\phi_{\bar{\tau}_{k-1}}^{1} \bar{S}_{\bar{\tau}_{k-1}}^{k}-\phi_{\bar{\tau}_{k}}^{1} \bar{S}_{\bar{\tau}_{k}}^{k}\right)=\sum_{k=1}^{n} \phi_{\bar{\tau}_{k-1}}^{1}\left(\bar{S}_{\bar{\tau}_{k-1}}^{k}-\bar{S}_{\bar{\tau}_{k-1}}^{k-1}\right)
$$

due to the condition that $\phi_{\bar{\tau}_{n}}^{1}=0$ as well as the standing assumption that $\phi_{\bar{\tau}_{0}}^{1}=\phi_{0}^{1}=0$. From the construction of $\bar{S}^{k}$, we already know that $\bar{S}_{\bar{\tau}_{k-1}}^{k}-\bar{S}_{\bar{\tau}_{k-1}}^{k-1} \geq 0, \mathbb{Q}^{k}$-a.s. and hence $\hat{\mathbb{Q}}^{k}$-a.s. from (3.20). Using the assumption that $\phi^{1}$ is nonpositive, we immediately obtain

$$
\mathbb{E}\left[\mathcal{E}\left(Y_{\bar{\tau}_{n}}\right) \sum_{k=1}^{n}\left(\phi_{\bar{\tau}_{k-1}}^{1} \bar{S}_{\bar{\tau}_{k-1}}^{k}-\phi_{\bar{\tau}_{k}}^{1} \bar{S}_{\bar{\tau}_{k}}^{k}\right)\right] \leq 0
$$

which yields that (3.22) holds and therefore (3.21) is verified.

Case 2: The portfolio process $\phi^{1}$ is a nonnegative process on $\llbracket 0, \bar{\tau}_{n} \rrbracket$. 
The proof follows the arguments in Case 1 with small modifications. We therefore only sketch some of the steps. We will mimic the idea in Case 1 for the construction of fictitious stock price processes. First, for $0 \leq t \leq \bar{\tau}_{1}$, we can define

$$
\ddot{S}_{t}^{1} \triangleq \tilde{S}_{t}^{1}
$$

For $\bar{\tau}_{1} \leq t \leq \bar{\tau}_{2}$, we first consider two auxiliary processes

$$
\begin{aligned}
& X_{t}^{\prime \prime} \triangleq \underset{\tau \in \mathcal{O}_{t}}{\operatorname{ess} \sup } \mathbb{E}^{\mathbb{Q}^{2}}\left[\breve{S}_{\tau}\left(1-\breve{\lambda}_{\tau}\right) \mid \mathcal{F}_{t}\right], \\
& Y_{t}^{\prime \prime} \triangleq \underset{\tau \in \mathcal{O}_{t}}{\operatorname{essinf}} \mathbb{E}^{\mathbb{Q}^{2}}\left[\breve{S}_{\tau}\left(1+\frac{\ddot{S}_{\bar{\tau}_{1}}^{1}-\breve{S}_{\bar{\tau}_{1}}}{\breve{S}_{\bar{\tau}_{1}} \breve{\lambda}_{\bar{\tau}_{1}}}\right) \mid \mathcal{F}_{t}\right],
\end{aligned}
$$

where $\mathcal{O}_{t}$ denotes the set of all stopping times $\tau$ with values $t \leq \tau \leq \bar{\tau}_{2}$. Let us further define

$$
X_{t} \triangleq \mathbb{E}^{\mathbb{Q}^{2}}\left[X_{\bar{\tau}_{1}}^{\prime \prime} \mid \mathcal{F}_{t}\right], \quad Y_{t} \triangleq \mathbb{E}^{\mathbb{Q}^{2}}\left[Y_{\bar{\tau}_{1}}^{\prime \prime} \mid \mathcal{F}_{t}\right]
$$

For $\bar{\tau}_{1} \leq t \leq \bar{\tau}_{2}$, it follows that $X_{t} \triangleq X_{t}^{\prime \prime}$ and $Y_{t} \triangleq Y_{t}^{\prime \prime}$. We then get that $(X)_{t \wedge \bar{\tau}_{1}}$ is a supermartingale and $(Y)_{t \wedge \bar{\tau}_{1}}$ is a submartingale under $\mathbb{Q}^{2}$. Moreover, similar to Case 1 , there exists a martingale $\ddot{M}^{2}$ under $\mathbb{Q}^{2}$ and $X_{t} \leq \ddot{M}_{t}^{2} \leq Y_{t}$ for $0 \leq t \leq \bar{\tau}_{2}$ and $\left(1-\breve{\lambda}_{t}\right) \breve{S}_{t} \leq \ddot{M}_{t}^{2} \leq\left(1+\breve{\lambda}_{t}\right) \breve{S}_{t}$ for $\bar{\tau}_{1} \leq t \leq \bar{\tau}_{2}$ as well as $\ddot{M}_{\bar{\tau}_{1}}^{2} \leq \ddot{S}_{\bar{\tau}_{1}}^{1}, \mathbb{Q}^{2}$-a.s.

Let us define the auxiliary stock price process for $0 \leq t \leq \bar{\tau}_{2}$ as

$$
\ddot{S}_{t}^{2} \triangleq\left\{\begin{aligned}
\tilde{S}_{t}^{2}, & \text { for } 0 \leq t<\bar{\tau}_{1} \\
\min \left(\tilde{S}_{t}^{2}, \ddot{M}_{t}^{2}\right), & \text { for } \bar{\tau}_{1} \leq t \leq \bar{\tau}_{2}
\end{aligned}\right.
$$

It is easy to check that $\ddot{S}^{2}$ is a supermartingale under $\mathbb{Q}^{2}$ for $0 \leq t \leq \bar{\tau}_{2}$ and $\left(1-\breve{\lambda}_{t}\right) \breve{S}_{t} \leq \ddot{S}_{t}^{2} \leq$ $\left(1+\breve{\lambda}_{t}\right) \breve{S}_{t}$ for $0 \leq t \leq \bar{\tau}_{2}$ and $\ddot{S}_{\bar{\tau}_{1}}^{2} \leq \ddot{S}_{\bar{\tau}_{1}}^{1}$. By repeating this procedure, we obtain a sequence of auxiliary processes $\left\{\ddot{S}^{k}\right\}_{1 \leq k \leq n}$ such that $\ddot{S}^{k}$ is a supermartingale under $\mathbb{Q}^{k}$ and $\left(1-\breve{\lambda}_{t}\right) \breve{S}_{t} \leq \ddot{S}_{t}^{k} \leq\left(1+\breve{\lambda}_{t}\right) \breve{S}_{t}$ for $0 \leq t \leq \bar{\tau}_{k}$. Moreover, we have the inequality $\ddot{S}_{\bar{\tau}_{k-1}}^{k} \leq \ddot{S}_{\bar{\tau}_{k-1}}^{k-1}, \mathbb{Q}^{k}$-a.s.

We will consider the fictitious semimartingale stock price process

$$
S_{t}^{\#} \triangleq \begin{cases}\ddot{S}_{t}^{k}, & \text { for } \bar{\tau}_{k-1} \leq t<\bar{\tau}_{k}, \quad k=1, \ldots, n \\ \ddot{S}_{\bar{\tau}_{n}}^{n}, & \text { for } t \geq \bar{\tau}_{n} .\end{cases}
$$

Eventually, similar to the proof in Case 1, it is enough to prove that

$$
\mathbb{E}^{\hat{\mathbb{Q}}^{n}}\left[\left(\phi^{1} \cdot S^{\sharp}\right)_{\bar{\tau}_{n}}\right]=\mathbb{E}\left[\mathcal{E}\left(Y_{\bar{\tau}_{n}}\right) \sum_{k=1}^{n}\left(\phi^{1} \mathbf{1}_{\rrbracket \bar{\tau}_{k-1}, \bar{\tau}_{k} \rrbracket} \cdot \ddot{S}^{k}\right)_{\bar{\tau}_{n}}+\mathcal{E}\left(Y_{\bar{\tau}_{n}}\right) \sum_{k=1}^{n}\left(\phi_{\bar{\tau}_{k-1}}^{1} \ddot{S}_{\bar{\tau}_{k-1}}^{k}-\phi_{\bar{\tau}_{k}}^{1} \ddot{S}_{\bar{\tau}_{k}}^{k}\right)\right] \leq 0 .
$$

But the inequality in (3.23) can be verified using the same proof as for (3.22) but replacing the submartingale with the supermartingale and using the facts that $\phi^{1}$ is nonnegative and $\ddot{S}_{\bar{\tau}_{k-1}}^{k} \leq \ddot{S}_{\bar{\tau}_{k-1}-1}^{k-1}$, $\mathbb{Q}^{k}$-a.s. and hence $\hat{\mathbb{Q}}^{k}$-a.s. for $1 \leq k \leq n$. 
Finally, we have shown that $\mathbb{E}^{\hat{Q}^{n}}[V] \leq 0$ for all $V \in \overline{\mathbf{C}}_{M}^{\bar{\tau}_{n}}(\breve{S}, \breve{\lambda})$. For the new sequence of probability measures $\left\{\hat{\mathbb{Q}}^{n}\right\}_{n \in \mathbb{N}}$, we can consider the special trading strategies defined in (3.17) and (3.18) again. As $\tilde{\phi}^{1}$ in (3.17) is nonpositive and $\hat{\phi}^{1}$ in (3.18) is nonnegative, it follows that

$$
\mathbb{E}^{\hat{\mathbb{Q}}^{n}}\left[V_{\bar{\tau}_{n}}^{\text {liq,0}}\left(\tilde{\phi}^{0}, \tilde{\phi}^{1}\right)\right] \leq 0, \quad \mathbb{E}^{\hat{\mathbb{Q}}^{n}}\left[V_{\bar{\tau}_{n}}^{\text {liq, } 0}\left(\hat{\phi}^{0}, \hat{\phi}^{1}\right)\right] \leq 0 .
$$

Following the proofs of lemmas 6.2 and 6.3 of Guasoni et al. (2012), we not only can obtain the existence of $\hat{S}_{t}^{n}$ for $0 \leq t \leq \bar{\tau}_{n}$ such that $\left(1-\breve{\lambda}_{t}\right) \breve{S}_{t} \leq \hat{S}_{t}^{n} \leq\left(1+\breve{\lambda}_{t}\right) \breve{S}_{t}$ and $\hat{S}^{n}$ is a martingale under $\hat{\mathbb{Q}}^{n}$ for $0 \leq t \leq \bar{\tau}_{n}$, but also get that $\hat{S}^{n}$ and $\hat{S}^{n+1}$ coincide $\mathbb{P}$-a.s. on $\llbracket 0, \bar{\tau}_{n} \rrbracket$ due to the fact that $\hat{\mathbb{Q}}^{n}=$ $\left.\hat{\mathbb{Q}}^{n+1}\right|_{\mathcal{F}_{\bar{\tau}_{n}}}$.

Recalling the definition of $\breve{S}$ and $\breve{\lambda}$ in (3.16), we obtain that for $0 \leq t \leq \bar{\tau}_{n}$,

$$
\begin{aligned}
\inf _{0 \leq t \leq \bar{\tau}_{n}}\left(S_{t}\left(1+\lambda_{t}\right)-\hat{S}_{t}^{n}\right) & >\inf _{0 \leq t \leq \bar{\tau}_{n}}\left(\frac{1}{2} S_{t}\left(1+\lambda_{t}\right)+\frac{1}{2} S_{t}^{\prime}\left(1+\lambda_{t}^{\prime}\right)-\hat{S}_{t}^{n}\right) \\
& =\inf _{0 \leq t \leq \bar{\tau}_{n}}\left(\breve{S}_{t}\left(1+\breve{\lambda}_{t}\right)-\hat{S}_{t}^{n}\right) \geq 0, \text { a.s. }
\end{aligned}
$$

Similarly, we can also check that

$$
\inf _{0 \leq t \leq \bar{\tau}_{n}}\left(\hat{S}_{t}^{n}-S_{t}\left(1-\lambda_{t}\right)\right)>0, \text { a.s. }
$$

To finish the proof, we will paste the processes $\left\{\hat{S}^{n}\right\}_{n \in \mathbb{N}}$ together over the whole horizon $[0, T]$ to get the process $\tilde{S}$. We claim that for the local martingale $Z$ defined in (3.19), the process $\tilde{S} Z$ is also a local martingale. To see this, for any stopping time $\tau$, we have $\tilde{S}_{0}=\mathbb{E}^{\hat{\mathbb{Q}}^{n}}\left[\tilde{S}_{\bar{\tau}_{n} \wedge \tau}\right]=\mathbb{E}\left[Z_{\bar{\tau}_{n}} \hat{S}_{\bar{\tau}_{n} \wedge \tau}\right]=$ $\mathbb{E}\left[Z_{\bar{\tau}_{n} \wedge \tau} \hat{S}_{\bar{\tau}_{n} \wedge \tau}\right]=\mathbb{E}\left[Z_{\bar{\tau}_{n} \wedge \tau} \tilde{S}_{\bar{\tau}_{n} \wedge \tau}\right]$ for all $n \in \mathbb{N}$. It follows that the process $\tilde{S} Z$ is a local martingale with the same localizing sequence $\left\{\bar{\tau}_{n}\right\}_{n \in \mathbb{N}}$ defined in (3.14).

\section{4 | EXAMPLES}

Two examples are constructed in this section, in which one stock price process is continuous and another stock price has jumps, in order to demonstrate that the existence of an SCLMS is weaker than the existence of a (S)CPS.

\subsection{The case of continuous stock price}

This example is essentially due to some of the results obtained in Rásonyi and Schachermayer (2010). We first provide a sufficient condition for the existence of an SCLMS that will be used in constructing the example. To this end, we shall first introduce the concept of obvious arbitrage (OA) in Rásonyi and Schachermayer (2010).

Definition 4.1. Let $S$ have continuous paths. We say that $S$ allows for an $O A$, if there are $\alpha>0$ and $[0, T] \cup\{+\infty\}$-valued stopping times $\sigma \leq \tau$ such that $\{\sigma<+\infty\}=\{\tau<+\infty\}, \mathbb{P}(\sigma<+\infty)>0$ and

$$
\begin{aligned}
\frac{S_{\tau}}{S_{\sigma}} \geq 1+\alpha, & \text { on }\{\sigma<+\infty\}, \\
\text { or } \frac{S_{\tau}}{S_{\sigma}} \leq \frac{1}{1+\alpha}, & \text { on }\{\sigma<+\infty\} .
\end{aligned}
$$


Proposition 4.2. Assume that the continuous stock price $S$ does not admit an OA. Then there exists $S C L M S$ with any constant transaction cost $\lambda \in(0,1)$, i.e., $\mathcal{Z}_{\text {loc }}(\lambda) \neq \emptyset$ for any constant $\lambda \in(0,1)$, and hence $\mathcal{Z}_{\text {loc }}^{s}(\lambda) \neq \emptyset$.

Proof. Proposition 1 of Rásonyi and Schachermayer (2010) proved that there exists a sequence of stopping times $\rho_{n}^{\lambda}$ such that the stopped process $S^{\rho_{n}^{\lambda}}$ admits a $\lambda$-CPS $\left(\tilde{S}^{n}, \mathbb{Q}^{n}\right)$. Moreover, according to their proof, one has the concatenation property; i.e., on each $\llbracket 0, \rho_{n-1}^{\lambda} \rrbracket$, we get that $\tilde{S}_{t}^{n}$ equals $\tilde{S}_{t}^{n-1}$ and $\left.\mathbb{Q}^{n-1}\right|_{\mathcal{F}_{\rho_{n-1}^{\lambda}}}=\left.\mathbb{Q}^{n}\right|_{\mathcal{F}_{\rho_{n-1}^{\lambda}}}$. Therefore, for each $n \in \mathbb{N}$, we can define $\mathbb{P}$-martingales $Z^{n}$ by

$$
Z_{T}^{n} \triangleq \frac{d\left(\mathbb{Q}^{n} \mid \mathcal{F}_{\rho_{n}^{\lambda}}\right)}{d\left(\mathbb{P} \mid \mathcal{F}_{\rho_{n}^{\lambda}}\right)}
$$

It is clear that $Z_{t}^{n}>0$; furthermore, we have $Z^{n}=Z^{n-1}$ on the stochastic interval $\llbracket 0, \rho_{n-1}^{\lambda} \rrbracket$. Therefore, by pasting the process $\left(Z^{n}\right)_{n \in \mathbb{N}}$, one can define a $\mathbb{P}$-local martingale such that $Z_{t}>0$ for all $t \in[0, T]$. Similarly, we can paste the process $\left(\tilde{S}^{n}\right)_{n \in \mathbb{N}}$. As $\tilde{S}^{n} Z^{n}$ is a $\mathbb{P}$-UI martingale according to the construction of each $\tilde{S}^{n}$, it is easy to see that $\tilde{S} Z$ is a P-local martingale. The existence of a CLMS $(\tilde{S}, Z)$ is then verified.

For any $\lambda \in(0,1)$, we can find a CLMS $\left(\tilde{S}^{\prime}, Z^{\prime}\right)$ for the stock price $S$ with smaller transaction cost $\lambda^{\prime} \in(0, \lambda)$ (by the above arguments). Clearly, $\left(\tilde{S}^{\prime}, Z^{\prime}\right)$ is a pair of SCLMS for the stock price $S$ with transaction cost $\lambda$ and $\mathcal{Z}_{\text {loc }}^{s} \neq \varnothing$.

Remark 4.3. We want to point out that the no OA condition is not necessary for the existence of SCLMS. The following example from Rásonyi (2015) illustrates this point: Define $X_{t} \triangleq \exp \left(W_{t}-\frac{t}{2}\right)$, $t \geq 0$ where $W_{t}$ is a Brownian motion and $\left(\mathcal{F}_{t}\right)_{t \geq 0}$ is its natural filtration. Define the a.s. finite stopping time

$$
\tau \triangleq \inf \left\{t: X_{t}=\frac{1}{2}\right\}
$$

and set

$$
S_{t}=X_{\tau \wedge \tan t}, \quad 0 \leq t<\frac{\pi}{2} ; \quad S_{\frac{\pi}{2}}=\frac{1}{2}
$$

Define also $\mathcal{G}_{t}=\mathcal{F}_{\tan t}, 0 \leq t<\frac{\pi}{2}$, and $\mathcal{G}_{\frac{\pi}{2}}=\mathcal{F}_{\infty}$. Clearly, the stock price process $S$ admits an $O A$ by setting $\sigma=0$ and $\tau=\frac{\pi}{2}$. However, the process $S_{t}$ is a $\mathcal{G}_{t}$-local martingale, proved by Prokaj and Rásonyi $(2011)$. We can see that $(\tilde{S}, \mathbb{Q}) \triangleq(S, \mathbb{P})$ is a pair of $\operatorname{SCPS}$ for any transaction cost $\lambda \in(0,1)$ and hence an SCLMS.

It is worth noting that the existence of an OA opportunity in this example is not a contradiction to the NA condition in Definition 2.5. Indeed, to take advantage of the OA opportunity, one will choose to short sell the stock $S$ at time $t=0$ and wait until time $\tau$ to buy it to cover the position. However, by the definitions of $S$ and $X$, this simple strategy is not admissible for any initial position $x>0$ as the liquidation value process may go to $-\infty$ at some stopping time $t<\tau$. Therefore, this market model still satisfies the usual NA condition of Definition 2.5 and there exists a pair of SCPS.

As an application of Proposition 4.2, we will demonstrate that an SCLMS might exist even when a CPS may not. 
Example 4.4. Let $\left(W_{t}\right)_{t \geq 0}$ be a standard Brownian motion with respect to $\left(\Omega, \mathcal{F}, \mathbb{P}^{0}\right)$, and define $X_{t}=$ $\exp \left(W_{t}-\frac{t}{2}\right)$. Define the sequence of stopping times $\left(\rho_{n}\right)_{n=1}^{\infty}$ by $\rho_{0}=0, \rho_{1}=\inf \left\{t \geq 0: X_{t}=2^{-2}\right.$ or 2$\}$ and, for $n \geq 1$, let

$$
\rho_{n+1}=\rho_{n} \mathbf{1}_{\left\{X_{\rho_{n}} \neq 2^{-2^{n}}\right\}}+\sigma_{n+1} \mathbf{1}_{\left\{X_{\rho_{n}}=2^{-2^{n}}\right\}},
$$

where

$$
\sigma_{n+1}=\inf \left\{t \geq 0: X_{t}=2^{-2^{n+1}} \text { or } 2^{-n+1}\right\}
$$

Define the stopping time

$$
\tau=\min \left\{\rho_{n}: X_{\rho_{n}}=2^{-n+2}\right\}
$$

and the stock price process $S$

$$
S_{t}=X_{t \wedge \tau}, \quad 0 \leq t<\infty
$$

Next, define a probability $\mathbb{P}$ on $\mathcal{F}$ by

$$
\frac{d \mathbb{P}}{d \mathbb{P}^{0}}=\sum_{n=1}^{\infty} \frac{2^{-n}}{\mathbb{P}^{0}\left(\tau=\rho^{n}\right)} \mathbf{1}_{\left\{\tau=\rho_{n}\right\}} .
$$

The market model consists of the price process $S$ under the probability $\mathbb{P}$. (One can then choose a deterministic time change from $[0,+\infty]$ to $[0, T]$ to turn this into a finite horizon model.) Proposition 7 of Rásonyi and Schachermayer (2010) proved that $\left(S_{t}\right)_{0 \leq t \leq T}$ satisfies the assumptions in Proposition 4.2. As a result, there exists an $\operatorname{SCLMS}(\tilde{S}, Z)$ with constant transaction cost $\lambda \in(0,1)$. However, they also showed that there is no CPS for the same transaction cost $\lambda \in(0,1)$. The argument is by contradiction: Here $\mathbb{P}$ is constructed so that $\mathbb{P}(\tau=\infty)=0$. However, if a CPS $(\tilde{S}, \mathbb{Q})$ exists, it would have to be that $\mathbb{Q}(\tau=\infty)>0$, which yields a contradiction.

\subsection{The case of jump process}

We will rely on the results of Ruf and Runggaldier (2013) to construct our example.

Example 4.5. Let $Y$ be a compensated $\mathbb{P}^{0}$-Poisson process with intensity $\beta=\frac{1}{T} \leq 1$ started from one, stopped when it hits zero or when it first jumps. Denote by $\tau$ the first hitting time of zero and by $\rho$ the first jump time. Set $S=Y$ and consider the constant transaction cost $\lambda \in(0,1)$. Then, $\mathbb{P}^{0}\left(Y_{T}=\right.$ $0)=\exp (-1)$. Let the initial wealth be $x=1-\exp (-1)$ and define the self-financing portfolio $\phi^{*}=$ $\left(\phi^{0, *}, \phi^{1, *}\right)$ by

$$
\phi_{t}^{1, *}=e^{-1+\beta t} \mathbf{1}_{\{t \leq \tau \wedge \rho\}}, \quad \phi_{t}^{0, *}=-\int_{0}^{t}(1+\lambda) S_{u} d \phi_{u}^{1, *}
$$

We can derive that

$$
\begin{aligned}
V_{T}^{l i q, x}\left(\phi^{0, *}, \phi^{1, *}\right) & =V_{\tau \wedge \rho}^{l i q, x}\left(\phi^{0, *}, \phi^{1, *}\right)=x+\phi_{\tau \wedge \rho}^{0, *}+\phi_{\tau \wedge \rho}^{1, *} S_{\tau \wedge \rho}-\lambda\left|\phi_{\tau \wedge \rho}^{1, *}\right| S_{\tau \wedge \rho} \\
& =x+\phi_{0}^{1, *} S_{0}+\int_{0}^{\tau \wedge \rho} \phi_{t}^{1, *} d S_{t}-\lambda \int_{0}^{\tau \wedge \rho} S_{t} d \phi_{t}^{1, *}-\lambda \phi_{\tau \wedge \rho}^{1, *} S_{\tau \wedge \rho},
\end{aligned}
$$


which can be computed explicitly as

$$
\begin{aligned}
V_{T}^{l i q, x}\left(\phi^{0, *}, \phi^{1, *}\right)= & x+e^{-1}+e^{-1+\beta \rho} \mathbf{1}_{\{\rho \leq \tau\}}-\beta \int_{0}^{\tau \wedge \rho} e^{-1+\beta t} d t-\lambda \int_{0}^{\tau \wedge \rho} S_{t} \beta e^{-1+\beta t} d t \\
& +\lambda(2-\beta \rho) e^{-1+\beta \rho} \mathbf{1}_{\{\rho \leq \tau\}}-\lambda e^{-1+\beta(\tau \wedge \rho)} S_{\tau \wedge \rho} \\
\geq & x+e^{-1}+(1+\lambda) e^{-1+\beta \rho} \mathbf{1}_{\{\rho \leq \tau\}}+\left(e^{-1}-e^{-1+\beta(\tau \wedge \rho)}\right) \\
& +2 \lambda\left(e^{-1}-e^{-1+\beta(\tau \wedge \rho)}\right)-2 \lambda e^{-1+\beta(\tau \wedge \rho)} \\
= & x+\left[(2+2 \lambda) e^{-1}-3 \lambda e^{-1+\beta \rho}\right] \mathbf{1}_{\{\rho \leq \tau\}}+\left[(2+2 \lambda) e^{-1}-(1+4 \lambda)\right] \mathbf{1}_{\{\tau<\rho\}} .
\end{aligned}
$$

The first inequality holds as $S_{t} \leq 2$ for any $t \in[0, T]$ and $\beta \rho \leq 1$ on $\{\rho \leq \tau\}$, and the last equality holds due to the fact that $\beta \tau=1$ on the event $\{\tau \leq \rho\}$. Let us choose $\lambda>0$ small enough such that

$$
x+(2+2 \lambda) e^{-1}-(1+4 \lambda) \geq 0
$$

which gives that $\lambda \leq \frac{1}{4 e-2}$. Then, we always have

$$
\left[x+(2+2 \lambda) e^{-1}-(1+4 \lambda)\right] \mathbf{1}_{\{\tau<\rho\}} \geq 0
$$

Recall that we have $\beta \rho \leq 1$ on $\{\rho \leq \tau\}$. With the choice of $\lambda \leq \frac{1}{4 e-2}$, it is easy to verify that on $\{\rho \leq \tau\}$, the following holds:

$$
x+(2+2 \lambda) e^{-1}-3 \lambda e^{-1+\beta \rho} \geq 1 .
$$

We obtain that if $\lambda \leq \frac{1}{4 e-2}$, then

$$
V_{T}^{l i q, x}\left(\phi^{0, *}, \phi^{1, *}\right) \geq \mathbf{1}_{\{\rho \leq \tau\}}=\mathbf{1}_{\left\{Y_{T}>0\right\}}, \text { a.s. }
$$

Moreover, for any $t<\tau \wedge \rho$, it is easy to see that

$$
\begin{aligned}
V_{t}^{l i q, x}\left(\phi^{0, *}, \phi^{1, *}\right) & =x-\int_{0}^{t}(1+\lambda)(1-\beta s) \beta e^{-1+\beta s} d s+(1-\lambda) e^{-1+\beta t}(1-\beta t) \\
& \geq x-(1+\lambda) t \beta \frac{1}{e} \geq x-(1+\lambda) \frac{1}{e}>0 \text { a.s. }
\end{aligned}
$$

because $(1-\beta t) e^{-1+\beta t}$ is decreasing in t and $1-\frac{1}{e}-(1+\lambda) \frac{1}{e}>0$ if $\lambda \leq \frac{1}{4 e-2}$. It is then verified that $\phi^{*}$ is $x$-admissible, i.e., $\phi^{*} \in \mathcal{A}_{x}$.

Let us define the probability $\mathbb{P}$ by

$$
\frac{d \mathbb{P}}{d \mathbb{P}^{0}}=Y_{T}
$$

The process $\frac{1}{Y}$ is a positive $\mathbb{P}$-strict local martingale with $\mathbb{P}\left(\frac{1}{Y_{T}}>0\right)=1$; see theorem 2.1 of Carr, Fisher, and Ruf (2014). Now, because the process $\frac{1}{Y}$ is a $\mathbb{P}$-local martingale and $\frac{S}{Y}=1$ is $a \mathbb{P}$ martingale, $(\tilde{S}, Z)=\left(S, \frac{1}{Y}\right)$ is an SCLMS. 
The nonexistence of a CPS can be proved by a contradiction argument. Let $(\tilde{S}, \mathbb{Q})$ be a CPS. For the fixed $x=1-e^{-1}$ and for any $\phi=\left(\phi^{0}, \phi^{1}\right) \in \mathcal{A}_{x}$, as in the proof of Proposition 3.1, we have

$$
0 \leq V_{T}^{l i q, x}\left(\phi^{0}, \phi^{1}\right) \leq x+\int_{0}^{T} \phi_{t}^{1} d \tilde{S}_{t}, \mathbb{P} \text {-a.s. }
$$

The local martingale property of $\tilde{S}$ under $\mathbb{Q}$ implies that $x+\int_{0}^{T} \phi_{t}^{1} d \tilde{S}_{t}$ is a supermartingale under the same measure. As a result,

$$
\mathbb{E}^{\mathbb{Q}}\left[V_{T}^{l i q, x}\left(\phi^{0}, \phi^{1}\right)\right] \leq x<1,
$$

for any $\phi \in \mathcal{A}_{x}$, which is now a contradiction to the fact that $V_{T}^{l i q, x}\left(\phi^{0, *}, \phi^{1, *}\right) \geq \mathbf{1}_{\left\{Y_{T}>0\right\}}=1$, P-a.s. (and hence $\mathbb{Q}$-a.s.).

\section{5 | UTILITY MAXIMIZATION PROBLEMS}

In this section, we will discuss the market viability property by showing the relationship between the existence of an SCLMS, the existence of an optimal solution to the utility maximization problem defined on the terminal liquidation value, and the existence of numéraire portfolios.

\section{$5.1 \mid$ Utility maximization problems}

We first show that the NUPBR and NLABP conditions in the robust sense are sufficient conditions on the market models for the market viability, which are generally weaker than the usual conditions in the existing literature. Some standard conditions on preferences are required.

Assumption 5.1. The utility function $U(\cdot)$ is defined on $(0, \infty)$ and $U(\cdot)$ is continuously differentiable, strictly increasing, and strictly concave. Without loss of generality, let us also assume $U(\infty)>0$. We further assume that the utility function satisfies the Inada conditions and the reasonable asymptotic elasticity, i.e.,

$$
U^{\prime}(0)=+\infty, U^{\prime}(\infty)=0, A E[U]=\limsup _{x \rightarrow \infty} \frac{x U^{\prime}(x)}{U(x)}<1
$$

The utility maximization problem on the terminal liquidation value process is defined by

$$
u(x)=\sup _{\left(\phi^{0}, \phi^{1}\right) \in \mathcal{A}_{x}(\lambda)} \mathbb{E}\left[U\left(V_{T}^{\mathrm{liq}, x}\left(\phi^{0}, \phi^{1}\right)\right)\right]=\sup _{V_{T}^{\mathrm{liq}, x} \in \mathcal{V}_{x}(\lambda)} \mathbb{E}\left[U\left(V_{T}^{\mathrm{liq}, x}\right)\right] .
$$

Due to the monotonicity of the function $U(\cdot)$, it follows that

$$
u(x)=\sup _{V_{T} \in \mathcal{C}(x)} \mathbb{E}\left[U\left(V_{T}\right)\right]
$$

where the convex solid set $\mathcal{C}(x)$ is defined in (3.4).

The next theorem is the second main result of this paper.

Theorem 5.2. Suppose that there exists some $x>0$ such that $u(x)<+\infty$ (and hence for all $x>0$ ). Consider the following three assertions: 
(1) S satisfies the NUPBR and NLABP conditions with the transaction cost $\lambda$ in the robust sense.

(2) For any initial wealth $x>0$, there exists a unique optimal portfolio $\left(\phi^{0, *}, \phi^{1, *}\right) \in \mathcal{A}_{x}(\lambda)$, i.e., $V_{T}^{*, x} \in \mathcal{V}_{x}(\lambda)$ such that

$$
u(x)=\mathbb{E}\left[U\left(V_{T}^{*, x}\right)\right] .
$$

(3) $S$ satisfies the NUPBR condition with the transaction cost $\lambda$.

We have the following implications: (1) $\Rightarrow(2) \Rightarrow(3)$.

Remark 5.3. As discussed in Section 3, under the assumption that $S$ satisfies the NUPBR and NLABP conditions with transaction cost $\lambda$, we may still have arbitrage opportunities in the sense of Definition 2.5. Theorem $5.2(1) \Rightarrow(2)$ states that as long as these arbitrage opportunities do not lead to UPBR or violate the NLABP condition, the optimal portfolio problem is still well defined. Either some types of arbitrages are not preferred by the investors, or they are too small or not scalable to result in infinitely large wealth.

Proof of (2) $\Rightarrow(3)$. To this end, let us prove that $S$ satisfies the NUPBR condition first.

Suppose that the utility maximization problem (5.1) admits an optimal solution for the market model with the stock price process $S$ and the transaction $\operatorname{cost} \lambda$. We need to check that the $\operatorname{set} \mathcal{V}_{1}(\lambda)$ is bounded in probability.

The conditions $\mathrm{AE}[U]<1$ and $U(\infty)>0$ yield the existence of constants $\alpha>0$ and $\gamma>0$ (see Kramkov \& Schachermayer, 1999) such that $U(\alpha)>0$ and

$$
x U^{\prime}(x)<\gamma U(x), \text { for all } x \geq \alpha .
$$

For any $V_{T}^{\text {liq, } 1} \in \mathcal{V}_{1}(\lambda)$, it is clear that $V_{T}^{\text {liq, } 1}+\alpha \in \mathcal{V}_{1+\alpha}(\lambda)$ as we can always keep the cash $\alpha>0$ in the riskless asset. Now, let us consider the investor with initial wealth $1+\alpha$, and assume that $V_{T}^{*, 1+\alpha}$ is optimal for the utility maximization problem

$$
\sup _{V_{T}^{\mathrm{liq}, 1+\alpha} \in \mathcal{V}_{1+\alpha}(\lambda)} \mathbb{E}\left[U\left(V_{T}^{\mathrm{liq}, 1+\alpha}\right)\right]=\mathbb{E}\left[U\left(V_{T}^{*, 1+\alpha}\right)\right]<\infty .
$$

For any $V_{T}^{\text {liq, } 1} \in \mathcal{V}_{1}(\lambda)$, we claim that $\left(V_{T}^{\text {liq, } 1}+\alpha-V_{T}^{*, 1+\alpha}\right) U^{\prime}\left(V_{T}^{*, 1+\alpha}\right)$ is integrable and in particular,

$$
\mathbb{E}\left[\left(V_{T}^{\mathrm{liq}, 1}+\alpha-V_{T}^{*, 1+\alpha}\right) U^{\prime}\left(V_{T}^{*, 1+\alpha}\right)\right] \leq 0 .
$$

We will first demonstrate this claim. To this end, for any fixed $\epsilon \in\left(0, \frac{1}{2}\right)$, we define $V_{T}^{\epsilon}=(1-$ $\epsilon) V_{T}^{*, 1+\alpha}+\epsilon\left(V_{T}^{\text {liq,1 }}+\alpha\right)$. Due to the convexity of the set $\mathcal{C}^{\lambda}(1+\alpha)$, we have $V_{T}^{\epsilon} \in C^{\lambda}(1+\alpha)$. The optimality of $V_{T}^{*, 1+\alpha}$ together with the concavity of $U(x)$ implies that

$$
\begin{aligned}
0 & \geq \frac{1}{\epsilon} \mathbb{E}\left[U\left(V_{T}^{\epsilon}\right)-U\left(V_{T}^{*, 1+\alpha}\right)\right] \geq \frac{1}{\epsilon} \mathbb{E}\left[\left(V_{T}^{\epsilon}-V_{T}^{*, 1+\alpha}\right) U^{\prime}\left(V_{T}^{\epsilon}\right)\right] \\
& =\mathbb{E}\left[\left(V_{T}^{\text {liq, } 1}+\alpha-V_{T}^{*, 1+\alpha}\right) U^{\prime}\left(V_{T}^{\epsilon}\right)\right],
\end{aligned}
$$

if we have that $\left(V_{T}^{\epsilon}-V_{T}^{*, 1+\alpha}\right) U^{\prime}\left(V_{T}^{\epsilon}\right)$ is integrable. Here, the second term in (5.4) is finite because $-\infty<U(\epsilon \alpha) \leq \mathbb{E}\left[U\left(V_{T}^{\epsilon}\right)\right]<\mathbb{E}\left[U\left(V_{T}^{*, 1+\alpha}\right)\right]<\infty$. For the third term, the concavity of $U(x)$ gives the 
upper bound $\left(V_{T}^{\epsilon}-V_{T}^{*, 1+\alpha}\right) U^{\prime}\left(V_{T}^{\epsilon}\right) \leq U\left(V_{T}^{\epsilon}\right)-U\left(V_{T}^{*, 1+\alpha}\right)$. Therefore, (5.4) holds if we can verify that the lower bound $-\left(V_{T}^{\epsilon}-V_{T}^{*, 1+\alpha}\right)^{-} U^{\prime}\left(V_{T}^{\epsilon}\right)$ is also integrable.

We show next that the family $\left\{\left(V_{T}^{\text {liq,1 }}+\alpha-V_{T}^{*, 1+\alpha}\right)^{-} U^{\prime}\left(V_{T}^{\epsilon}\right), \epsilon \in\left(0, \frac{1}{2}\right)\right\}$ is dominated by an integrable random variable. Let us write

$$
\begin{aligned}
\left(V_{T}^{\mathrm{liq}, 1}+\alpha-V_{T}^{*, 1+\alpha}\right)^{-} U^{\prime}\left(V_{T}^{\epsilon}\right) & =\left(V_{T}^{\mathrm{liq}, 1}+\alpha-V_{T}^{*, 1+\alpha}\right)^{-} U^{\prime}\left(V_{T}^{\epsilon}\right) \mathbf{1}_{\left\{V_{T}^{*, 1+\alpha} \leq \alpha\right\}} \\
& +\left(V_{T}^{\mathrm{liq}, 1}+\alpha-V_{T}^{*, 1+\alpha}\right)^{-} U^{\prime}\left(V_{T}^{\epsilon}\right) \mathbf{1}_{\left\{V_{T}^{*, 1+\alpha} \geq 2 \alpha\right\}} \\
& +\left(V_{T}^{\mathrm{liq}, 1}+\alpha-V_{T}^{*, 1+\alpha}\right)^{-} U^{\prime}\left(V_{T}^{\epsilon}\right) \mathbf{1}_{\left\{\alpha<V_{T}^{*, 1+\alpha}<2 \alpha\right\}}
\end{aligned}
$$

For the first term in (5.5), we can see that

$$
\left(V_{T}^{\text {liq, } 1}+\alpha-V_{T}^{*, 1+\alpha}\right)^{-} U^{\prime}\left(V_{T}^{\epsilon}\right) \mathbf{1}_{\left\{V_{T}^{*, 1+\alpha} \leq \alpha\right\}}=0 \text {, a.s. }
$$

For the second term in (5.5), we obtain an estimate by the monotonicity of $U^{\prime}(x)$ and $U(x)$ and (5.2):

$$
\begin{aligned}
\left(V_{T}^{\mathrm{liq}, 1}+\alpha-V_{T}^{*, 1+\alpha}\right)^{-} & U^{\prime}\left(V_{T}^{\epsilon}\right) \mathbf{1}_{\left\{V_{T}^{*, 1+\alpha} \geq 2 \alpha\right\}} \\
& \leq\left(V_{T}^{\mathrm{liq}, 1}+\alpha-V_{T}^{*, 1+\alpha}\right)^{-} U^{\prime}\left((1-\epsilon) V_{T}^{*, 1+\alpha}\right) \mathbf{1}_{\left\{V_{T}^{*, 1+\alpha} \geq 2 \alpha\right\}} \\
& \leq\left(V_{T}^{\mathrm{liq}, 1}+\alpha-V_{T}^{*, 1+\alpha}\right)^{-} U^{\prime}\left(\frac{1}{2} V_{T}^{*, 1+\alpha}\right) \mathbf{1}_{\left\{V_{T}^{*, 1+\alpha} \geq 2 \alpha\right\}} \\
& \leq \gamma \frac{V_{T}^{*, 1+\alpha}}{\frac{1}{2} V_{T}^{*, 1+\alpha}} U\left(\frac{1}{2} V_{T}^{*, 1+\alpha}\right) \mathbf{1}_{\left\{V_{T}^{*, 1+\alpha} \geq 2 \alpha\right\}} \\
& \leq 2 \gamma U^{+}\left(\frac{1}{2} V_{T}^{*, 1+\alpha}\right) \leq 2 \gamma U^{+}\left(V_{T}^{*, 1+\alpha}\right) .
\end{aligned}
$$

The right-hand side is integrable as we know that $u(1+\alpha)=\mathbb{E}\left[U\left(V_{T}^{*, 1+\alpha}\right)\right]<\infty$.

For the last term in (5.5), again, by the monotonicity of $U^{\prime}(x)$, we obtain

$$
\begin{aligned}
\left(V_{T}^{\mathrm{liq}, 1}+\alpha-V_{T}^{*, 1+\alpha}\right)^{-} & U^{\prime}\left(V_{T}^{\epsilon}\right) \mathbf{1}_{\left\{\alpha<V_{T}^{* 1+\alpha}<2 \alpha\right\}} \\
& \leq\left(V_{T}^{\mathrm{liq}, 1}+\alpha-V_{T}^{*, 1+\alpha}\right)^{-} U^{\prime}\left(\frac{1}{2} V_{T}^{*, 1+\alpha}\right) \mathbf{1}_{\left\{\alpha<V_{T}^{*, 1+\alpha}<2 \alpha\right\}} \\
& \leq V_{T}^{*, 1+\alpha} U^{\prime}\left(\frac{1}{2} V_{T}^{*, 1+\alpha}\right) \mathbf{1}_{\left\{\alpha<V_{T}^{*, 1+\alpha}<2 \alpha\right\}} \\
& \leq 2 \alpha U^{\prime}\left(\frac{1}{2} \alpha\right)<\infty .
\end{aligned}
$$


Hence, we can conclude that $\left\{\left(V_{T}^{\text {liq, } 1}+\alpha-V_{T}^{*, 1+\alpha}\right)^{-} U^{\prime}\left(V_{T}^{\epsilon}\right), \epsilon \in\left(0, \frac{1}{2}\right)\right\}$ is bounded above by a nonnegative integrable random variable, which we will denote by $\Gamma$, and hence $\mathbb{E}\left[\left(V_{T}^{\epsilon}-V_{T}^{*, 1+\alpha}\right) U^{\prime}\left(V_{T}^{\epsilon}\right)\right]>$ $-\infty$ and the inequality (5.4) is verified. Applying Fatou's lemma

$$
\mathbb{E}\left[\left(V_{T}^{\mathrm{liq}, 1}+\alpha-V_{T}^{*, 1+\alpha}\right) U^{\prime}\left(V_{T}^{*, 1+\alpha}\right)\right] \leq \liminf _{\epsilon \rightarrow 0} \mathbb{E}\left[\left(V_{T}^{\mathrm{liq}, 1}+\alpha-V_{T}^{*, 1+\alpha}\right) U^{\prime}\left(V_{T}^{\epsilon}\right)\right] \leq 0,
$$

where we used the facts that $\left(V_{T}^{\text {liq, } 1}+\alpha-V_{T}^{*, 1+\alpha}\right) U^{\prime}\left(V_{T}^{\epsilon}\right) \geq-\left(V_{T}^{\text {liq,1 }}+\alpha-V_{T}^{*, 1+\alpha}\right)^{-} U^{\prime}\left(V_{T}^{\epsilon}\right) \geq-\Gamma$ and that $\Gamma$ is a nonnegative integrable random variable. Equation (5.3) holds as a consequence.

Because $\left(V_{T}^{\text {liq, } 1}+\alpha-V_{T}^{*, 1+\alpha}\right) U^{\prime}\left(V_{T}^{*, 1+\alpha}\right)$ is integrable for any $V_{T}^{\text {liq, } 1}$, by taking the special case $V_{T}^{\text {liq,1 }}=0$ as we are allowed to throw away cash, we conclude that $\left(V_{T}^{*, 1+\alpha}-\alpha\right) U^{\prime}\left(V_{T}^{*, 1+\alpha}\right)$ is also integrable. Therefore, it follows from (5.3) and the concavity of $U$ that

$$
\begin{aligned}
\sup _{V_{T}^{\text {liq, }, 1} \in \mathcal{V}_{1}(\lambda)} \mathbb{E}\left[V_{T}^{\text {liq,1 }} U^{\prime}\left(V_{T}^{*, 1+\alpha}\right)\right] & \leq \mathbb{E}\left[\left(V_{T}^{*, 1+\alpha}-\alpha\right) U^{\prime}\left(V_{T}^{*, 1+\alpha}\right)\right] \\
& \leq \mathbb{E}\left[U\left(V_{T}^{*, 1+\alpha}\right)\right]-U(\alpha)<\infty .
\end{aligned}
$$

If we can show $U^{\prime}\left(V_{T}^{*, 1+\alpha}\right)>0$ a.s., then by lemma 3.2 of Imkeller and Perkowski (2015), we can conclude that the set $\mathcal{V}_{1}(\lambda)$ is bounded in probability. We will prove this by a contradiction argument and assume $\mathbb{P}\left(U^{\prime}\left(V_{T}^{*, 1+\alpha}\right)=0\right)>0$. Consider the two cases:

Case 1: If $U(\infty)=\infty$.

It is easy to get $\mathbb{P}\left(U\left(V_{T}^{*, 1+\alpha}\right)=\infty\right)>0$ as $U^{\prime}(\infty)=0$. We obtained a contradiction to the fact that $u(1+\alpha)=\mathbb{E}\left[U\left(V_{T}^{*, 1+\alpha}\right)\right]<\infty$.

Case 2: If $0<U(\infty)<\infty$.

We only get that $\mathbb{P}(A)>0$ for $A \triangleq\left\{V_{T}^{*, 1+\alpha}=\infty\right\}$. Using (5.2), we obtain that

$$
\mathbb{E}\left[U^{\prime}\left(V_{T}^{*, 1+\alpha}\right) \mathbf{1}_{A}\right]<\gamma \mathbb{E}\left[\frac{U\left(V_{T}^{*, 1+\alpha}\right)}{V_{T}^{*, 1+\alpha}} \mathbf{1}_{A}\right] .
$$

But the fact that $U^{\prime}(\infty)=0$ leads to $\mathbb{E}\left[U^{\prime}\left(V_{T}^{*, 1+\alpha}\right) \mathbf{1}_{A}\right]=0$. For the right-hand side, we know that $0<U(\infty)<\infty$ and therefore $\mathbb{E}\left[\frac{U\left(V_{T}^{*, 1+\alpha}\right)}{V_{T}^{*, 1+\alpha}} \mathbf{1}_{A}\right]=0$, which is a contradiction to the strict inequality.

In conclusion, we deduce that $\mathbb{P}\left(U^{\prime}\left(V_{T}^{*, 1+\alpha}\right)>0\right)=1$, which completes the proof of the implication (2) $\Rightarrow$ (3); i.e., $S$ satisfies the NUPBR condition with the transaction $\operatorname{cost} \lambda$.

Proof of (1) $\Rightarrow(2)$. We first build the bipolarity result for the set $\mathcal{C}(x)$. Let us first define the polar of this set:

$$
\mathcal{Y}(y)=(\mathcal{C}(x))^{\circ}=\left\{Y_{T} \in \mathbb{L}_{+}^{0}: Y_{0}=y \quad \text { and } \quad \mathbb{E}\left[V_{T} Y_{T}\right] \leq x y, \forall V_{T} \in \mathcal{C}(x)\right\} .
$$

As $S$ satisfies the NUPBR and NLABP conditions with the transaction cost $\lambda$ in the robust sense, Theorem 2.8 gives the existence of the $\operatorname{SCLMS}(\tilde{S}, Z)$. Following verbatim the proof of $(3.1)$ for the pair $(S, \lambda)$ instead of $\left(S^{\prime}, \lambda^{\prime}\right)$, we can obtain that

$$
\sup _{V_{T}^{\mathrm{liq}, x} \in \mathcal{V}_{x}(\lambda ; S)} \mathbb{E}\left[V_{T}^{\mathrm{liq}, x} Z_{T}\right] \leq x,
$$


which implies that

$$
\mathcal{M} \triangleq\left\{Z_{T} \in \mathbb{L}_{+}^{0}:(\tilde{S}, Z) \in \mathcal{Z}_{\text {loc }}^{s}\right\} \subseteq \mathcal{Y}(1) .
$$

Hence, we conclude that $\mathcal{Y}(1)$ is not empty as $\mathcal{M}$ is not empty. Clearly, we have $\mathcal{Y}(y)=y \mathcal{Y}(1)$ and $\mathcal{Y}(1)=(\mathcal{C}(1))^{\circ}$. Moreover, because $\mathcal{C}(1)$ is convex, solid, and closed under the convergence in probability (due to Proposition 3.3), we have that

$$
\mathcal{C}(1)=(\mathcal{Y}(1))^{\circ}, \quad \text { and } \quad \mathcal{Y}(1)=(\mathcal{C}(1))^{\circ},
$$

due to the bipolar theorem of Brannath and Schachermayer (1999). Due to (5.7), we also have that $\mathcal{C}(1)$ is bounded in probability because $\mathcal{V}_{1}$ is bounded in probability, and that it contains the constant 1 . Therefore, it is clear that the constant $x \in \mathcal{C}(x)$ and we have $\mathcal{Y}(y) \subseteq \mathbb{L}^{1}$. Now we can apply theorem 3.1 and theorem 3.2 of Kramkov and Schachermayer (1999) to conclude that for each $y>0$, there exists an optimal solution $Y_{T}^{*}(y)$ to the dual optimization problem

$$
v(y)=\inf _{Y \in \mathcal{Y}(y)} \mathbb{E}\left[V\left(Y_{T}\right)\right],
$$

and we have a conjugate duality between the primal and dual value functions

$$
v(y)=\sup _{x>0}[u(x)-x y], \quad y>0 ; \quad u(x)=\inf _{y>0}[v(y)+x y], \quad x>0 .
$$

Moreover, the unique optimal solution $V_{T}^{*, x}$ to the utility maximization problem is given by

$$
V_{T}^{*, x}=I\left(Y_{T}^{*}(y)\right)
$$

where $y=u^{\prime}(x)$ and $\mathbb{E}\left[V_{T}^{*, x} Y_{T}^{*}(y)\right]=x y$.

\subsection{Existence of numéraire portfolios}

Here, we briefly discuss the existence of a numéraire portfolio and some other related concepts as a corollary of Theorem 5.2 and Proposition 3.3. We first define some relevant notions.

Definition 5.4. A liquidation value process $V \in \mathcal{V}_{1}(\lambda)$ is called

(i) a numéraire portfolio, denoted by $V^{\text {num }}$, if

$$
\mathbb{E}\left[\frac{V_{T}^{l i q, 1}}{V_{T}}\right] \leq 1 ;
$$

(ii) a log-optimal portfolio, denoted by $V^{\log }$, if

$$
\mathbb{E}\left[\log \left(V_{T}^{l i q, 1}\right)\right] \leq \mathbb{E}\left[\log \left(V_{T}\right)\right]
$$

(iii) a growth-optimal or relatively log-optimal portfolio, denoted by $V^{g o p}$, if

$$
\mathbb{E}\left[\log \left(\frac{V_{T}^{l i q, 1}}{V_{T}}\right)\right] \leq 0,
$$

for all $V^{l i q, 1} \in \mathcal{V}_{1}(\lambda)$ 
Corollary 5.5. Consider the following assertions:

(1) $S$ satisfies the NUPBR and NLABP conditions with the transaction cost $\lambda$.

(2) The numéraire portfolio $V^{\text {num }}$ for $S$ with the transaction cost $\lambda$ exists and $V_{T}^{\text {num }}<+\infty$ a.s.

(3) The growth-optimal portfolio $V^{\text {gop }}$ for $S$ with the transaction cost $\lambda$ exists and $V_{T}^{\text {gop }}<+\infty$ a.s.

(4) $S$ satisfies the NUPBR condition with the transaction cost $\lambda$.

(5) The log-optimal portfolio $V^{\log }$ for $S$ with the transaction cost $\lambda$ exists.

We have implications (1) $\Rightarrow(2) \Leftrightarrow(3) \Rightarrow$ (4). Moreover, if $u(x)<\infty$ in (5.1) with $U(x)=\log (x)$, we have the equivalence $(2) \Leftrightarrow(3) \Leftrightarrow(5)$ and $V_{T}^{\log }=V_{T}^{\text {gop }}=V_{T}^{\text {num }}$.

Proof of (1) $\Rightarrow$ (2). The proof follows the line of arguments presented in the proof of theorem 5.1 of Christensen and Larsen (2007). We provide this for the sake of completeness. Consider the functions $f_{n}(x)$ defined by

$$
f_{n}(x) \triangleq \log (x) \mathbf{1}_{\{x \leq n\}}+g_{n}(x) \mathbf{1}_{\{x>n\}},
$$

where $g_{n}$ is bounded, concave such that $f_{n}$ is two times continuous differentiable satisfying the Inada conditions and $g_{n}^{\prime}$ is a convex function less than $\frac{1}{x}$. Clearly, $f_{n}(x) \rightarrow \log (x)$ as $n \rightarrow \infty$ for all $x>0$.

According to our Theorem 5.2, if $S$ satisfies the NUPBR and NLABP conditions in the robust sense, there exists a unique optimal solution $V^{*, n}$ of the following utility maximization problem:

$$
\sup _{V_{T}^{\text {liq }} \in \mathcal{C}(1)} \mathbb{E}\left[f_{n}\left(V_{T}^{\mathrm{liq}}\right)\right]
$$

By choosing the forward convex combination $\tilde{V}^{n} \in \operatorname{conv}\left\{V^{*, n}, V^{*, n+1}, \ldots\right\}$ and passing to the subsequence if necessary, we can assume that $\tilde{V}^{n}$ converges almost surely to some $V^{*}$. Moreover, because $\mathcal{C}(1)$ is closed and bounded in probability, we have $V^{*} \in \mathcal{C}(1)$ and $V^{*}<+\infty$ a.s. Notice that $f_{n}^{\prime}(x) \leq \frac{1}{x}$ and $f_{n}^{\prime}(x) \rightarrow \frac{1}{x}$ for all $x$. We obtain that

$$
\mathbb{E}\left[\frac{V_{T}^{\mathrm{liq}}}{V_{T}^{*}}\right] \leq \liminf _{n \rightarrow \infty} \mathbb{E}\left[V_{T}^{\mathrm{liq}} f_{n}^{\prime}\left(\tilde{V}_{T}^{n}\right)\right]
$$

Let us assume that $V_{T}^{\text {liq }}$ is bounded. Recalling that $\tilde{V}^{n}=\sum_{k=n}^{\infty} \theta_{k} V^{*, k}$, we get

$$
\begin{aligned}
\mathbb{E}\left[V_{T}^{\mathrm{liq}} f_{n}^{\prime}\left(\tilde{V}_{T}^{n}\right)\right] & \leq \mathbb{E}\left[V_{T}^{\mathrm{liq}} \sum_{k=n}^{\infty} \theta_{k} f_{n}^{\prime}\left(V_{T}^{*, k}\right)\right] \\
& =\mathbb{E}\left[V_{T}^{\mathrm{liq}} \sum_{k=n}^{\infty} \theta_{k} f_{k}^{\prime}\left(V_{T}^{*, k}\right)\right]+\mathbb{E}\left[V_{T}^{\mathrm{liq}} \sum_{k=n}^{\infty} \theta_{k}\left(f_{n}^{\prime}\left(V_{T}^{*, k}\right)-f_{k}^{\prime}\left(V_{T}^{*, k}\right)\right)\right] .
\end{aligned}
$$

For the first term, it is easy to see from the proof of Theorem 5.2 that

$$
\mathbb{E}\left[V_{T}^{\text {liq }} \sum_{k=n}^{\infty} \theta_{k} f_{k}^{\prime}\left(V_{T}^{*, k}\right)\right]=\sum_{k=n}^{\infty} \theta_{k} \mathbb{E}\left[V_{T}^{\text {liq }} f_{k}^{\prime}\left(V_{T}^{*, k}\right)\right] \leq \sum_{k=n}^{\infty} \theta_{k} \mathbb{E}\left[V_{T}^{*, k} f_{k}^{\prime}\left(V_{T}^{*, k}\right)\right] \leq 1 .
$$


It follows that

$$
\begin{aligned}
\mathbb{E}\left[V_{T}^{\mathrm{liq}} f_{n}^{\prime}\left(\tilde{V}_{T}^{n}\right)\right] & \leq 1+\mathbb{E}\left[V_{T}^{\mathrm{liq}} \sum_{k=n}^{\infty} \theta_{k}\left(f_{n}^{\prime}\left(V_{T}^{*, k}\right)-f_{k}^{\prime}\left(V_{T}^{*, k}\right)\right)\right] \\
& =1+\mathbb{E}\left[V_{T}^{\mathrm{liq}} \sum_{k=n}^{\infty} \theta_{k}\left(f_{n}^{\prime}\left(V_{T}^{*, k}\right)-f_{k}^{\prime}\left(V_{T}^{*, k}\right)\right) \mathbf{1}_{\left\{V_{T}^{*, k} \geq n\right\}}\right] \\
& \leq 1+\mathbb{E}\left[V_{T}^{\mathrm{liq}} \sum_{k=n}^{\infty} \theta_{k} \frac{1}{V_{T}^{*, k}} \mathbf{1}_{\left\{V_{T}^{*, k} \geq n\right\}}\right] \leq 1+\frac{\mathbb{E}\left[V_{T}^{\mathrm{liq}}\right]}{n},
\end{aligned}
$$

from which it follows that $\mathbb{E}\left[\frac{V_{T}^{\text {liq }}}{V_{T}^{*}}\right] \leq 1$. When $V_{T}^{\text {liq }}$ is not bounded, we can prove the same result for $V_{T}^{\text {liq, } M}=V_{T}^{\text {liq }} \wedge M$ for $M>0$, and then apply the monotone convergence theorem to get the same conclusion. Hence, we proved the existence of a numéraire portfolio $V^{\text {num }}=V^{*}$ and $V_{T}^{\text {num }}<+\infty$ a.s.

Proof of (2) $\Rightarrow$ (3). This follows by an application of Jensen's inequality, and by setting $V^{\text {gop }}=V^{\text {num }}$, we get

$$
\mathbb{E}\left[\log \left(\frac{V_{T}^{\text {liq }}}{V_{T}^{\text {gop }}}\right)\right] \leq \log \mathbb{E}\left[\frac{V_{T}^{\text {liq }}}{V_{T}^{\text {gop }}}\right] \leq 0
$$

Proof of $(3) \Rightarrow(2)$. The existence of $V_{T}^{\text {gop }}$ implies that

$$
0 \leq \mathbb{E}\left[\log V_{T}^{\text {gop }}-\log V_{T}^{\text {liq }}\right]
$$

for all $V_{T}^{\text {liq }} \in \mathcal{V}_{1}$. For each fixed $V_{T}^{\text {liq }} \in \mathcal{V}_{1}$, define $V_{T}^{\epsilon}=(1-\epsilon) V_{T}^{\text {gop }}+\epsilon V_{T}^{\text {liq }} \in \mathcal{C}(1)$. As $1+\log (x) \leq$ $x$, we obtain

$$
0 \leq \mathbb{E}\left[\log V_{T}^{\mathrm{gop}}-\log V_{T}^{\epsilon}\right] \leq \mathbb{E}\left[\frac{V_{T}^{\mathrm{gop}}-V_{T}^{\epsilon}}{V_{T}^{\epsilon}}\right]=\epsilon \mathbb{E}\left[\frac{V_{T}^{\mathrm{gop}}-V_{T}^{\mathrm{liq}}}{V_{T}^{\epsilon}}\right]
$$

Therefore, we derive that

$$
\mathbb{E}\left[\frac{V_{T}^{\mathrm{liq}}}{V_{T}^{\epsilon}}\right] \leq \mathbb{E}\left[\frac{V_{T}^{\mathrm{gop}}}{V_{T}^{\epsilon}}\right]
$$

Observing that for $\epsilon<\frac{1}{2}$, we have

$$
\frac{V_{T}^{\mathrm{liq}}-V_{T}^{\mathrm{gop}}}{V_{T}^{\epsilon}} \geq-2
$$

we apply Fatou's lemma to (5.10) to obtain

$$
\mathbb{E}\left[\frac{V_{T}^{\text {liq }}}{V_{T}^{\text {gop }}}\right] \leq 1
$$


Proof of (2) $\Rightarrow$ (4). Let $V_{T}^{\text {num }} \in \mathcal{V}_{1}$ be such that $\sup _{V_{T}^{\text {liq }} \in \mathcal{V}_{1}} \mathbb{E}\left[\frac{V_{T}^{\text {liq }}}{V_{T}^{\text {num }}}\right] \leq 1$ and $V_{T}^{\text {num }}<+\infty$ a.s. Clearly, $\frac{1}{V_{T}^{\text {num }}}>0$ a.s. As a result, it is clear that $\mathcal{V}_{1}$ is bounded in probability, and hence $S$ satisfies NUPBR with transaction cost $\lambda$.

Under the additional assumption that $u(x)<\infty$ with $U(x)=\log x$, the proof of (2) $\Leftrightarrow$ (5) follows almost exactly the proof of proposition 4.3 of Becherer (2001).

\section{6 | ADDITIONAL DISCUSSIONS}

\subsection{A discussion on no arbitrage conditions}

Based on our proofs in Section 3, we aim to briefly discuss different types of arbitrage opportunities in market models with transaction costs and compare them to the ones in the frictionless case.

First of all, to distinguish the major difference between our paper and the literature on market viability in frictionless markets, it is worth noting that the NUPBR condition in Karatzas and Kardaras (2007) implies the NLABP condition in market models without transactions. To wit, it is well known that the NUPBR condition is equivalent to the existence of a local martingale deflator $Y$. Given the localizing sequence $\left\{\tau_{n}\right\}_{n \in \mathbb{N}}$ for $Y$, we obtain the equivalent local martingale measures $\mathbb{Q}^{n}$ on $\llbracket 0, \tau_{n} \rrbracket$. The fundamental theorem of asset pricing in Delbaen and Schachermayer (1994) asserts that the market model satisfies the NA condition locally on each $\llbracket 0, \tau_{n} \rrbracket$. Therefore, we always have (NUPBR) $\Rightarrow(\mathrm{NLA}) \Rightarrow$ (NLABP). However, in our setting, the NUPBR condition in Definition 2.3 and the NLABP condition in Definition 2.4 may not imply each other. This special feature caused by transaction costs is the main motivation of this paper.

Second, it is also of interest to examine some of the conclusions in Delbaen and Schachermayer (1995) using the cost value process $V^{\text {cost, } x}$ defined in (3.11). In particular, instead of the two kinds of arbitrages discussed in lemma 3.1 in Delbaen and Schachermayer (1995), there are three different kinds of arbitrages in our setting with transaction costs. To compare different notions of arbitrage opportunities, it is actually difficult to use our NLABP condition in Definition 2.4 that requires NA locally for a sequence of stopping times. In other words, the opposite of NLABP is too abstract to describe. To this end, we shall consider the following stronger notion of arbitrage.

Definition 6.1. We say that $S$ admits an LA with the transaction cost $\lambda$ if there exists a stopping time $\tau$ (we only consider stopping times valued in $[0, T]$ in this paper) with $\mathbb{P}(\tau<T)>0$ and an admissible portfolio $\left(\phi^{0}, \phi^{1}\right) \in \mathcal{A}(\lambda)$ such that,

$$
\mathbb{P}\left(V_{\tau}^{\text {liq, },}\left(\phi^{0}, \phi^{1}\right) \geq 0\right)=1, \quad \text { and } \quad \mathbb{P}\left(V_{\tau}^{\text {liq, }, 0}\left(\phi^{0}, \phi^{1}\right)>0 \mid \tau<T\right)>0 .
$$

If we cannot find such a stopping time and portfolio, we say that the stock price process $S$ satisfies the strong-NLA condition under the transaction cost $\lambda$.

It follows that the strong-NLA condition implies the NLABP condition in Definition 2.4.

For the rest of our discussion, let us recall lemma 3.1 of Delbaen and Schachermayer (1995) (a slightly modified version) from the infinite horizon to the finite horizon.

Lemma 6.2. If the càdlàg semimartingale $S$ admits an arbitrage with respect to general admissible integrands, then there is an $S$-integrable strategy $H$ satisfying either of the following:

(i) $(H \cdot S)$ is nonnegative and the arbitrage is scalable. 
(ii) $H$ is 1 -admissible and there exist $\epsilon>0$ and a stopping time $\tau$ with $\mathbb{P}(\tau<T)>0$ such that $H=$ $H \mathbf{1}_{\rrbracket \tau, T \rrbracket}$ and $(H \cdot S)_{T} \geq \epsilon$ on the set $\{\tau<T\}$.

From the proof in Delbaen and Schachermayer (1995), the case (ii) corresponds to the scenario in which the process $(H \cdot S)$ becomes negative with positive probability. It is clear that a scalable arbitrage (SA) in case (i) is an UPBR, whereas case (ii) describes a more conventional form of arbitrage. An LA may happen in case (ii).

A special example in case (i) is called an immediate arbitrage (IA) as defined below (see also definition 3.2 of Delbaen and Schachermayer, 1995).

Definition 6.3. In frictionless market models, we say that the semimartingale $S$ admits an IA at the stopping time $\tau$ where $\mathbb{P}(\tau<T)>0$ if there exists an $S$-integrable strategy $H$ such that $H=H \mathbf{1}_{\rrbracket \tau, T \rrbracket}$ and $(H \cdot S)_{t}>0$ for $t>\tau \mathbb{P}$ a.s.

It is easy to see that the IA implies an UPBR on the terminal wealth because $H$ is scalable and the sequence $H^{n} \triangleq n H$ for $n \in \mathbb{N}$ leads to an UPBR. Therefore, the no immediate arbitrage (NIA) condition is closely related to the NUPBR condition defined in Karatzas and Kardaras (2007) in frictionless markets.

In the presence of transaction costs, the notion of IA becomes more delicate because when the investor wants to take advantage of this arbitrage opportunity and enter the portfolio position at time $\tau$ and liquidate it immediately after $\tau$, the transaction cost $2 \lambda_{\tau} S_{\tau}\left|\phi_{\tau}^{1}\right|$ has to be paid. Therefore, we cannot define the IA simply by identifying the sign of the liquidation value process. In fact, we need to impose conditions on both the liquidation value process and the cost value process.

Definition 6.4. We say that $S$ admits an IA at the stopping time $\tau$ if $\mathbb{P}(\tau<T)>0$ and there exists a portfolio $\left(\phi^{0}, \phi^{1}\right)$ such that $V_{\tau}^{\text {cost }, 0}\left(\phi^{0}, \phi^{1}\right)<V_{t}^{\text {liq, }, 0}\left(\phi^{0}, \phi^{1}\right)$ on $\rrbracket \tau, T \rrbracket$.

Therefore, there must be a jump size of at least $2 \lambda_{\tau} S_{\tau}\left|\phi_{\tau}^{1}\right|$ at the stopping time $\tau$ for the emergence of an IA. The following result is a simple observation based on Definition 6.4.

Unlike the discussion in Lemma 6.2, which is simply based on whether the arbitrage wealth process is negative or not, the types of arbitrages we consider depend on the delicate comparison between the liquidation value process and the cost value process. For any admissible arbitrage portfolio $\left(\phi^{0}, \phi^{1}\right)$ (in the sense of Definition 2.5), one of the following holds:

(1) We have $V_{t}^{\text {liq, }, 0}\left(\phi^{0}, \phi^{1}\right) \geq 0$ for all $t \in[0, T]$.

(2) There exists some stopping time $\tau<T$ such that $\mathbb{P}\left(V_{\tau}^{\text {liq, }, 0}\left(\phi^{0}, \phi^{1}\right)<0\right)>0$. Two subcases may occur:

(a) There exists some $[0, T]$-valued stopping times $s_{1}$ and $s_{2}$ with $\mathbb{P}\left(s_{1}<s_{2}<T\right)>0$ such that $V_{s_{1}}^{\text {cost }, 0}\left(\phi^{0}, \phi^{1}\right) \leq V_{s_{2}}^{\text {liq }, 0}\left(\phi^{0}, \phi^{1}\right)$ on the set $\left\{s_{1}<s_{2}<T\right\}$ and $\mathbb{P}\left(V_{s_{1}}^{\text {cost, } 0}\left(\phi^{0}, \phi^{1}\right)<\right.$ $\left.V_{s_{2}}^{\text {liq, } 0}\left(\phi^{0}, \phi^{1}\right) \mid s_{1}<s_{2}<T\right)>0$.

(b) For any $[0, T]$-valued stopping times $s_{1}$, $s_{2}$ with $\mathbb{P}\left(s_{1}<s_{2}<T\right)>0$, we have $\mathbb{P}\left(V_{s_{1}}^{\text {cost }, 0}\left(\phi^{0}, \phi^{1}\right)>V_{s_{2}}^{\text {liq }, 0}\left(\phi^{0}, \phi^{1}\right) \mid s_{1}<s_{2}<T\right)>0 \quad$ or $\quad V_{s_{1}}^{\text {cost }, 0}\left(\phi^{0}, \phi^{1}\right)=V_{s_{2}}^{\text {liq, } 0}\left(\phi^{0}, \phi^{1}\right)$ on the set $\left\{s_{1}<s_{2}<T\right\}$.

We obtain the following categorization of arbitrage opportunities based on the comparison between $V^{\text {liq,0 }}$ and $V^{\text {cost, } 0}$.

Lemma 6.5. If there is an arbitrage in the sense of Definition 2.5, then there is a self-financing portfolio $\left(\phi^{0}, \phi^{1}\right)$ satisfying one of the following: 
(i) $V_{t}^{\text {liq, },}\left(\phi^{0}, \phi^{1}\right) \geq 0$ for $t \in[0, T]$ (and therefore the arbitrage is scalable).

(ii) There exists two $[0, T]$-valued stopping times $\tau_{1}$ and $\tau_{2}$ with $\mathbb{P}\left(\tau_{1}<\tau_{2}<T\right)>0$ such that $\phi^{1}$ is supported on $\rrbracket \tau_{1}, \tau_{2} \rrbracket$ with $\mathbb{P}\left(V_{\tau_{2}}^{\text {liq, }}\left(\phi^{0}, \phi^{1}\right)>0 \mid \tau_{2}<T\right)>0$ and $\mathbb{P}\left(V_{\tau_{2}}^{\text {liq,0 }}\left(\phi^{0}, \phi^{1}\right) \geq 0\right)=1$ (and therefore it is an $L A$ ).

(iii) For any $[0, T]$-valued stopping time $\tau$ such that $\mathbb{P}(\tau<T)>0$, we either have $\mathbb{P}\left(V_{\tau}^{\text {liq, }, 0}\left(\phi^{0}, \phi^{1}\right)<\right.$ $0 \mid \tau<T)>0$ or $V_{\tau}^{\text {liq, }, 0}\left(\phi^{0}, \phi^{1}\right)=0$ on the set $\{\tau<T\}$ (and therefore it is neither an SA nor an $L A)$.

Proof. Clearly, (1) and (i) are equivalent.

When (2)(a) holds, there exists stopping times $s_{1}$ and $s_{2}$ with $\mathbb{P}\left(s_{1}<s_{2}<T\right)>0$ such that $V_{s_{1}}^{\text {cost }, 0}\left(\phi^{0}, \phi^{1}\right) \leq V_{s_{2}}^{\text {liq, } 0}\left(\phi^{0}, \phi^{1}\right)$ on the set $\left\{s_{1}<s_{2}<T\right\}$ and $\mathbb{P}\left(V_{s_{1}}^{\text {cost }, 0}\left(\phi^{0}, \phi^{1}\right)<V_{s_{2}}^{\text {liq, } 0}\left(\phi^{0}, \phi^{1}\right) \mid s_{1}<\right.$ $\left.s_{2}<T\right)>0$. Define the stopping time $\tau_{1} \triangleq s_{1}$ and $\tau_{2} \triangleq s_{2}$. We consider the following portfolio:

$$
\begin{aligned}
& \hat{\phi}_{t}^{1}=\phi_{t}^{1} \mathbf{1}_{\rrbracket \tau_{1}, \tau_{2} \rrbracket}, \\
& \hat{\phi}_{t}^{0}=\left(\phi_{t}^{0}-V_{\tau_{1}}^{\mathrm{cost}, 0}\left(\phi^{0}, \phi^{1}\right)\right) \mathbf{1}_{\rrbracket \tau_{1}, \tau_{2} \rrbracket}+\left(V_{\tau_{2}}^{\mathrm{liq}, 0}\left(\phi^{0}, \phi^{1}\right)-V_{\tau_{1}}^{\mathrm{cost}, 0}\left(\phi^{0}, \phi^{1}\right)\right) \mathbf{1}_{\rrbracket \tau_{2}, T \rrbracket} .
\end{aligned}
$$

It is easy to check that $\left(\hat{\phi}^{0}, \hat{\phi}^{1}\right)$ satisfies (ii).

When (2)(b) holds and there exists an LA, we obtain a contradiction when $s_{1}=0$ and $V_{0}^{\text {cost, } 0}\left(\phi^{0}, \phi^{1}\right)=0$. Therefore, (iii) is satisfied.

Remark 6.6. It is easy to observe that the SA in case (i) is an UPBR and (ii) corresponds to an LA opportunity. Let (TA) be the type of arbitrage that only happens at the terminal time $T$ as in statement (iii). As a result, we have identified

$$
(\text { Arbitrage })=(S A) \cup(L A) \cup(T A) .
$$

Consequently, we have

$$
(N A)=(N S A) \cap(\text { Strong }-N L A) \cap(N T A),
$$

and

$$
(N U P B R) \Rightarrow N S A,
$$

as well as

$$
\text { (Strong-NLA) } \Rightarrow N L A B P \text {. }
$$

Comparing with Lemma 6.2 reveals interesting differences between types of arbitrages (including the definition of an IA) between our paper and Delbaen and Schachermayer (1995). Recall that NFLVR $=N A+N U B P R$ and that in the case without transaction costs, NUBPR is enough for the existence of a local martingale deflator (see, e.g., Karatzas \& Kardaras, 2007). The NLABP condition is required in our main result due to the special and more complicated structures of arbitrage opportunities. Also, the trading size of $\left|\phi^{1}\right|$ has an important impact on the total transaction amount that the investor needs to pay; therefore, the arbitrage argument in our setting relies heavily on the condition that $\left|\phi^{1}\right|$ is bounded or not. (See Lemma 3.8 for the mathematical reasons behind this.) In the end, as stated in Section 3, the existence of SCLMS is equivalent to both the NUPBR and NLABP conditions in the robust sense. 


\subsection{About admissibility for utility maximization problems}

Let us now switch back to the discussion on utility maximization problems in Section 5 . We briefly discuss the reason why we should choose $x$-admissible portfolios as in (2.2) and require the NUPBR and NLABP conditions using only $x$-admissible portfolios.

To start, note that the utility maximization problem can still be well defined for a larger set of admissible portfolios.

Definition 6.7. For any $x \geq 0$, the self-financing portfolio $\left(\phi^{0}, \phi^{1}\right)$ is called tolerable if the liquidation value process satisfies

$$
V_{T}^{l i q, x}\left(\phi^{0}, \phi^{1}\right) \triangleq x+\phi_{T}^{0}+\left(\phi_{T}^{1}\right)^{+}\left(1-\lambda_{T}\right) S_{T}-\left(\phi_{T}^{1}\right)^{-}\left(1+\lambda_{T}\right) S_{T} \geq 0 .
$$

Let $\mathcal{A}_{x}^{\text {tol }}$ denote the set of all $x$-tolerable portfolios and $\mathcal{A}^{\text {tol }}=\bigcup_{x \geq 0} \mathcal{A}_{x}^{\text {tol }}$.

It is clear that $\mathcal{A}_{x} \subset \mathcal{A}_{x}^{\text {tol }}$. We introduce the definition of tolerable portfolios because, for any initial wealth $x>0$, the utility maximization problem on nonnegative terminal liquidation values is well defined on the set $\mathcal{A}_{x}^{\text {tol }}$ even if the portfolio $\left(\phi^{0}, \phi^{1}\right)$ is not $x$-admissible. Consider

$$
w(x)=\sup _{\left(\phi^{0}, \phi^{1}\right) \in \mathcal{A}_{x}^{\mathrm{tol}}} \mathbb{E}\left[U\left(V_{T}^{\mathrm{liq}, x}\left(\phi^{0}, \phi^{1}\right)\right)\right] .
$$

It is possible that $w(x)<\infty$ for some $x>0$ and that the optimization problem (6.3) admits a unique optimal solution. The natural question is whether we can discuss the market viability property for utility maximization problems defined using $x$-tolerable portfolios. The answer is negative in general. Although the value function $w(x)<\infty$ is well defined, to obtain the market viability using the dual characterization, the bipolar relationship between the appropriate primal and the dual sets and the closedness property of the primal set are essential. These properties may not hold for $x$-tolerable portfolios. Indeed, if we do not require the portfolio liquidation process to be nonnegative for all $t \in[0, T]$ as in Definition 6.7 and modify the NUPBR and NLABP conditions using the enlarged set $\mathcal{A}_{x}^{\text {tol }}$, we are actually making much stronger assumptions on market models as we have the obvious implications

$$
\mathrm{NUPBR}-\mathcal{A}_{x}^{\mathrm{tol}} \Rightarrow \mathrm{NUPBR}-\mathcal{A}_{x} \text { and NLABP }-\mathcal{A}^{\mathrm{tol}} \Rightarrow \mathrm{NLABP}-\mathcal{A} \text {. }
$$

Moreover, the SCLMS or even the SCPS is no longer the necessary dual element for the primal set of liquidation value processes. To see this, let $(\tilde{S}, \mathbb{Q})$ be a pair of SCPS. We already know that

$$
V_{T}^{\text {liq }, x}\left(\phi^{0}, \phi^{1}\right) \leq \int_{0}^{T} \phi_{t}^{1} d \tilde{S}_{t}, \quad \mathbb{P} \text {-a.s. }
$$

for some self-financing portfolio $\left(\phi^{0}, \phi^{1}\right)$. Assuming that $\left(\phi^{0}, \phi^{1}\right) \in \mathcal{A}_{x}^{\text {tol }}$, we obtain that $M_{t} \triangleq x+$ $\int_{0}^{t} \phi_{u}^{1} d \tilde{S}_{u}$ is a local martingale under $\mathbb{Q}$. We only know that $M_{T} \geq 0$. Hence, the local martingale $M_{t}$ is not necessarily a supermartingale and it is difficult to verify the dual characterization

$$
\mathbb{E}^{\mathbb{Q}}\left[V_{T}^{\mathrm{liq}, x}\right] \leq \mathbb{E}^{\mathbb{Q}}\left[M_{T}\right] \leq x
$$

To guarantee market viability for $x$-tolerable portfolios, we have to introduce some artificial dual elements $Y$ such that

$$
\mathbb{E}\left[V_{T}^{\mathrm{liq}, x}\left(\phi^{0}, \phi^{1}\right) Y_{T}\right] \leq x, \quad \forall\left(\phi^{0}, \phi^{1}\right) \in \mathcal{A}_{x}^{\mathrm{tol}} .
$$


Unfortunately, it is in general difficult to provide the probabilistic characterization of the artificial dual element $Y$. As a result, it is impossible to make reasonable assumptions that would guarantee the existence of $Y$ and the market viability for $x$-tolerable portfolios. One way to reconcile this is to restrict the set $\mathcal{A}_{x}$ to a reasonable set of working portfolio so that SCLMS and SPCS can still serve as the dual elements. For example, we can allow the liquidation value processes to be bounded below by some stochastic process instead of a uniform constant. See the definition of $x$-acceptable portfolios in market models with transaction costs in Bayraktar and $\mathrm{Yu}$ (2015) and $\mathrm{Yu}$ (2017).

In conclusion, although the utility maximization problems may be well defined for $x$-tolerable portfolios, to make the market viability property mathematically tractable, it is reasonable to restrict the attention to the smaller set of $x$-admissible portfolios as we did in the previous sections.

\section{ACKNOWLEDGMENTS}

We thank two anonymous referees and the associate editor for their careful reviews, which led to a much improved paper.

\section{REFERENCES}

Ansel, J. P., \& Stricker, C. (1994). Couverture des actifs contingents et prix maximum. Annales Institute Henri Poincaré Probability and Statistics, 30(2), 303-315.

Bayraktar, E., \& Sayit, H. (2010). On the stickiness property. Quantitative Finance, 10(10), 1109-1112.

Bayraktar, E., \& Yu, X. (2015). Optimal investment with random endowments and transaction costs: Duality theory and shadow prices. Availabe at SSRN: https://ssrn.com/abstract=2779119

Becherer, D. (2001). The numeraire portfolio for unbounded semimartingales. Finance and Stochastics, 5(3), 327-341.

Brannath, W., \& Schachermayer, W. (1999). A bipolar theorem for $L_{+}^{0}(\Omega, \mathcal{F}, \mathbb{P})$. In J., Azéma et al. (Eds.), Séminaire de Probabilités, XXXIII. Lecture Notes in Mathematics (Vol. 1709, pp. 349-354). Berlin: Springer.

Carr, P., Fisher, T., \& Ruf, J. (2014). On the hedging of options on exploding exchange rates. Finance and Stochastics, 18(1), 115-144.

Choulli, T., Deng, J., \& Ma, J. (2015). How non-arbitrage, viability and numéraire portfolio are related. Finance and Stochastics, 19(4), 719-741.

Christensen, M. M., \& Larsen, K. (2007). No arbitrage and the growth optimal portfolio. Stochastic Analysis and Applications, 25(1), 255-280.

Cvitanić, J., \& Karatzas, I. (1996). Hedging and portfolio optimization under transaction costs: A martingale approach. Mathematical Finance, 6(2), 133-165.

Delbaen, F., \& Schachermayer, W. (1994). A general version of the fundamental theorem of asset pricing. Mathematische Annalen, 300(3), 463-520.

Delbaen, F., \& Schachermayer, W. (1995). The existence of absolutely continuous local martingale measures. Annals of Applied Probability, 5(4), 926-945.

Guasoni, P. (2002). Optimal investment with transaction costs and without semimartingales. Annals of Applied Probability, 12(4), 1227-1246.

Guasoni, P., Lépinette, E., \& Rásonyi, M. (2012). The fundamental theorem of asset pricing under transaction costs. Finance and Stochastics, 16(4), 741-777.

Guasoni, P., Rásonyi, M., \& Schachermayer, W. (2008). Consistent price systems and face-lifting pricing under transaction costs. Annals of Applied Probability, 18(2), 491-520.

Imkeller, P., \& Perkowski, N. (2015). The existence of dominating local martingale measures. Finance and Stochastics, 19(4), 685-717.

Jouini, E., \& Kallal, H. (1995). Martingales and arbitrage in securities markets with transaction costs. Journal of Economic Theory, 66(1), 178-197. 
Kabanov, Y., \& Safarian, M. (2009). Markets with transaction costs: Mathematical theory. Springer Finance. Berlin: Springer-Verlag.

Karatzas, I., \& Kardaras, C. (2007). The numéraire portfolio in semimartingale financial models. Finance and Stochastics, 11(4), 447-493.

Kramkov, D., \& Schachermayer, W. (1999). The asymptotic elasticity of utility functions and optimal investment in incomplete markets. Annals of Applied Probability, 9(3), 904-950.

Maris, F., Mbakop, E., \& Sayit, H. (2011). Consistent price systems for bounded processes. Communications on Stochastic Analysis, 5(4), 633-645.

Prokaj, V., \& Rásonyi, M. (2011). Local and true martingales in discrete time. Theory of Probability and its Applications, 325-332. (Translated from Teoriya Veroyatnoster̆ieë Primeneniya, 55(2), 398-404 (2010)).

Rásonyi, P. G. M. (2015). Fragility of arbitrage and bubbles in diffusion models. Finance and Stochastics, 19(2), $215-231$.

Rásonyi, P. G. M., \& Schachermayer, W. (2010). The fundamental theorem of asset pricing for continuous processes under small transaction costs. Annals of Finance, 6(2), 157-191.

Ruf, J., \& Runggaldier, W. (2013). A systematic approach to constructing market models with arbitrage. Arbitrage, Credit and Information Risks. Proceeding of the Sino-French Research Program in Financial Mathematics Conference, Beijing.

Sayit, H., \& Viens, F. (2011). Arbitrage-free models in markets with transaction costs. Electronic Communications in Probability, 16, 614-622.

Schachermayer, W. (2015). Admissible trading strategies under transaction costs. In C., Donati-Martin, A. Lejay, \& A., Rouault (Eds.), Seminaire de Probabilite XLVI. Lecture Notes in Mathematics (Vol. 2123, pp. 317-331). Berlin: Springer.

Takaoka, K., \& Schweizer, M. (2014). A note on the condition of no unbounded profit with bounded risk. Finance and Stochastics, 18(2), 393-405.

Yu, X. (2017). Optimal consumption under habit formation in markets with transaction costs and random endowments. Annals of Applied Probability, 27(2), 960-1002.

How to cite this article: Bayraktar E, Yu X. On the market viability under proportional transaction costs. Mathematical Finance. 2018;28:800-838. https://doi.org/10.1111/mafi.12155 